\title{
Multiple View Geometry of General Algebraic Curves *
}

\author{
J.Y. Kaminski ${ }^{1}$ and Amnon Shashua ${ }^{2}$ \\ ${ }^{1}$ Bar-Ilan University, \\ Mathematics and Computer Science Department, \\ Ramat-Gan, Israel. \\ 2 The Hebrew University Of Jerusalem, \\ School Of Computer Science and Engineering, \\ Jerusalem, Israel.
}

\begin{abstract}
We introduce a number of new results in the context of multiview geometry from general algebraic curves. We start with the recovery of camera geometry from matching curves. We first show how one can compute, without any knowledge on the camera, the homography induced by a single planar curve. Then we continue with the derivation of the extended Kruppa's equations which are responsible for describing the epipolar constraint of two projections of a general algebraic curve. As part of the derivation of those constraints we address the issue of dimension analysis and as a result establish the minimal number of algebraic curves required for a solution of the epipolar geometry as a function of their degree and genus.

We then establish new results on the reconstruction of general algebraic curves from multiple views. We address three different representations of curves: (i) the regular point representation in which we show that the reconstruction from two views of a curve of degree $d$ admits two solutions, one of degree $d$ and the other of degree $d(d-1)$. Moreover using this representation, we address the problem of homography recovery for planar curves. (ii) dual space representation (tangents) for which we derive a lower bound for the number of views necessary for reconstruction as a function of the curve degree and genus, and (iii) a new representation (to computer vision) based on the set of lines meeting the curve which does not require any curve fitting in image space, for which we also derive lower bounds for the number of views necessary for reconstruction as a function of curve degree alone.
\end{abstract}

${ }^{*}$ This work is partially supported by the Emmy Noether Institute for Mathematics and the Minerva Foundation of Germany, by the Excellency Center of the Israel Science Foundation "Group Theoretic Methods in the Study of Algebraic Varieties" and by EAGER (European network in Algebraic Geometry).

\section{Introduction}

A large body of research has been devoted to the problem of analyzing the 3D structure of the scene from multiple views. The necessary multi-view theory is by now well understood when the scene consists of point and line features - a summary of the past decade of work in this area can be found in $[20,10]$ and references to earlier work in [9].

The theory is somewhat fragmented when it comes to curve features, especially non-planar algebraic curves of general degree. Given known projection matrices [35, 29, $30]$ show how to recover the $3 \mathrm{D}$ position of a conic section from two and three views, and [37] show how to recover the homography matrix of the conic plane, and $[16,41]$ shows how to recover a quadric surface from projections of its occluding conics.

Reconstruction of higher-order curves were addressed in [26, 25, 4, 33, 34]. In [4] the matching curves are represented parametrically where the goal is to find a reparameterization of each matching curve such that in the new parameterization the points traced on each curve are matching points. The optimization is over a discrete parameterization, thus, for a planar algebraic curve of degree $n$, which is represented by $\frac{1}{2} n(n+3)$ points, one would need $n(n+3)$ minimal number of parameters to solve for in a non-linear bundle adjustment machinery - with some prior knowledge of a good initial guess. In [33, 34] the reconstruction is done under infinitesimal motion assumption with the computation of spatio-temporal derivatives that minimize a set of non-linear equations at many different points along the curve. In [25] only planar algebraic curves were considered, whereas in [26], the plane of non-planar algebraic curve $3 \mathrm{D}$ reconstruction is addressed.

On the problem of recovering the camera geometry (projection matrices, epipolar geometry, multi-view tensors) from matching projections of algebraic curves, the literature is sparse. [23, 25] show how to recover the fundamental matrix from matching conics with the result that 4 match- 
ing conics are minimally necessary for a unique solution. [25] generalize this result to higher order curves, but consider only planar curves and [26] generalizes this result to non-planar curves.

In this paper we address the general issue of multi-view geometry of algebraic curves from both angles: (i) recovering camera geometry (fundamental matrix or homography in the case of planar curve), and (ii) reconstruction of the curve from its projections across two or more views. Short versions of this work were published in $[25,26]$.

The necessary mathematical background is rather broad and the reader is referred to the Appendix for a formal mathematical introduction and to Section 2 for an informal introduction to the main introductory concepts. In Section 3 we start with the derivation of the extended Kruppa's equations which govern the epipolar constraint of two projections of a general algebraic curve. We first address the case of planar curves. In that context, we also show how to recover the homography generated by a single planar curve. Then we turn our attention to the general case. Furthermore as part of the derivation of the extended Kruppa's equations in the general case, we address the issue of dimension analysis and as a result establish the minimal number of algebraic curves required for a solution of the epipolar geometry as a function of their degree and genus.

On the reconstruction front (section 4), we address three different representations of curves: (i) the regular point representation for which we show that two views of a curve of degree $d$ admit two solutions, one of degree $d$ and the other of degree $d(d-1)$ (moreover, using this representation, we address the problem of homography matrix recovery for planar curves), (ii) dual space representation (image measurements are tangent lines) for which we derive a formula for the minimal number of views necessary for reconstruction as a function of the curve degree and genus, and (iii) a new representation (with regard to computer vision) based on the set of lines meeting the curve which does not require any curve fitting in image space, for which we also derive formulas for the minimal number of views necessary for reconstruction as a function of curve degree alone.

For the latter two representations we also address the problem of recovering a curved trajectory from a moving camera (as in [38] for conics). We derive formulas which specify the minimal number of views necessary for reconstruction as a function of curve degree and/or genus.

Beyond the technical contributions of this paper, our work paves the way to introducing a more powerful language, based on algebraic geometry, for handling curved objects for which the standard linear projective tools which have become popular in the computer vision literature become a particular case. The standard tool of projective geometry allow the consideration of points and lines as fundamental features, whereas with the tools of algebraic ge- ometry one can introduce higher level features with higher configurational complexity.

\section{Informal Mathematical Back- ground}

\subsection{Algebraic varieties}

The central concept to be used in this paper is the definition of an affine variety. This is the locus of common zeros of a family of polynomials. More precisely, consider a family of polynomials in $n$ variables. The locus of their common zeros defines an algebraic variety in the $n$-dimensional affine space. When the polynomials are all homogeneous, this variety can also be regarded as being embedded in the $n-1$-dimensional projective space, and is called a projective variety.

A variety can be formed as the union of subvarieties, and when these subvarieties are independent they are called the components of the variety. The variety is said to be irreducible if it has only one component (the variety itself).

The dimension of a variety can be defined in several different ways. The topological definition of dimension is the supremum of the lengths of chains of embedded irreducible components. However it can been proven that this definition is equivalent to the intuitive concept of dimension which is the number of independent parameters necessary to describe the variety.

\subsection{Relation between affine and projective va- rieties}

As mentioned above, an affine varieties is defined by a set of polynomials equations in $n$ variables. Such a set defines an affine variety in the $n$-dimensional affine space. On the other hand to define projective varieties, we need homogeneous polynomials. Consider a set of homogeneous polynomials in $n+1$ variables. This set defines a projective variety in the $n$-dimensional projective space. However one can also consider this set defines an affine variety in the $(n+1)$-dimensional affine space. This latter variety is called the affine cone over the former projective variety.

There exists also another relation, of major importance, between affine and projective varieties. Consider a projective variety, say $V_{p} \subset \mathbb{P}^{n}$, defined by homogeneous equations $f_{i}\left(X_{1}, \ldots, X_{n+1}\right)=0$. If the hyperplane at infinity in $\mathbb{P}^{n}$ is defined by $x_{n+1}=0$, then variety defined by $f_{i}\left(X_{1}, \ldots, X_{n}, 1\right)=0$ is an affine variety, say $V_{a}$, included in $n$-dimensional affine space. $V_{p}$ is called the projective closure of $V_{a} . V_{a}$ can be regarded as the affine piece of $V_{p}$ and the varieties defined by $f_{i}\left(X_{1}, \ldots, X_{n}, 0\right)=0$ is made of the points infinity of $V_{p}$. 


\subsection{Complex or real varieties}

When dealing with applications, the varieties can also defined by equations with real coefficients. However algebraic varieties are well behaved when regarded as complex varieties. So we shall consider all of them as varieties defined by equations with complex coefficients. This allows using all the power of algebraic geometry. For the computations, however, after the results is obtained over the complex numbers, we eventually consider only the real solutions.

\subsection{Algebraic Planar Curves}

A curve is a one-dimensional variety. The particular case of planar and spatial curves is of special interest for us. When embedded in a projective plane, a curve is the zero locus of a single homogeneous polynomial in three variables. Let $f$ be such a polynomial, then $f$ can factored as follows: $f=\prod f_{i}$, where $f_{i}$ are irreducible polynomials. In this case, the components of the curve are simply the subcurves defined by $f_{i}=0$. In order to avoid multiple components, we will consider only the case where $f$ is square-free, that is when $f_{i} \neq f_{j}$ for $i \neq j$. Note that when the polynomial $f$ is irreducible then the curve is an irreducible variety in the sense defined above.

The degree of a planar curve is simply the degree of its defining polynomial. To better understand the geometric meaning of this notion, consider a curve $\mathcal{C}$ embedded in the projective plane defined by $f(x, y, z)=0$. Let $\mathcal{L}$ be a line generated by two points $\mathbf{a}$ and $\mathbf{b}$. A point $\mathbf{p}=\mathbf{a}+\lambda \mathbf{b}$ on $\mathcal{L}$ is located on the curve if $J(\lambda)=f(\mathbf{a}+\lambda \mathbf{b})$ has a root. Since the degree of $J$, as function of $\lambda$, is the degree of $f$, the degree of $\mathcal{C}$ is the number of points a general line in the plane meets the curve.

Now suppose that the point $\mathbf{a}$ is on the curve $\mathcal{C}$. Consider then a first order Taylor expansion of $J(\lambda)$. We get: $J(\lambda)=$ $f(\mathbf{a})+\lambda \nabla f(\mathbf{a})^{T} \mathbf{b}$. Then two cases must be considered. If $\nabla f(\mathbf{a})=\mathbf{0}$, the point $\mathbf{a}$ is called a singular point of the curve $\mathcal{C}$. Otherwise the point is said to be regular or simple. We shall say that the line $\mathcal{L}$ is tangent to the curve $\mathcal{C}$ at a if a is regular and $\lambda=0$ is a double root of $J(\lambda)$.

An important concept is the multiplicity of a singularity. Consider a singular point a of $\mathcal{C}$, then the multiplicity of a is the smallest integer $m$ such that there exists a triple of integers $(i, j, k)$ such that $i+j+k=m$ and

$$
\frac{\partial^{m} f}{\partial^{i} x \partial^{j} y \partial^{k} z} \neq 0 .
$$

The concept of tangent can be generalized so that a point of multiplicity $m$ has $m$ tangents. We shall say that a multiple point is ordinary if all its tangents are distinct. An ordinary multiple point of multiplicity 2 is called a node. It has two distinct tangent.

A planar algebraic curve, say $\mathcal{C}$, has a natural dual object. The set of tangents to simple points of the curve is also an algebraic curve embedded in the dual plane. It is called the dual curve. Hence there exists a polynomial $\phi(u, v, w)$ such that $(u, v, w)$ is a zero of $\phi$ if it represents the coordinates of line tangent to $\mathcal{C}$ at a simple point. The degree of $\mathcal{C}$ and it dual curve $\mathcal{C}^{*}$ are easily related when the only singular points of $\mathcal{C}$ are nodes. In that case the degree of the dual curve $m$ is:

$$
m=d(d-1)-2(\# \text { nodes }),
$$

where $d$ is the degree of $\mathcal{C}$ and (\#nodes) is the number of nodes.

Consider once again the function $J(\lambda)$ defined above. When the Taylor expansion of $J$ vanishes up to third order, the tangent line $\mathcal{L}$ intersects the curve $\mathcal{C}$ at three coincident points. In that case, the tangency point is said to be an inflexion point. It turns out that inflexion points can be recovered with the Hessian curve (see Appendix for further details).

Finally a topological invariant of the curve can be defined. It is called the genus of the curve. When the only singularities of the curve are nodes and the degree of the curve is $d$, the genus $g$ is given by:

$$
g=\frac{(d-1)(d-2)}{2}-(\# \text { nodes })
$$

where (\#nodes) is the number of nodes as defined above.

\subsection{Algebraic Spatial Curves}

A spatial algebraic curve is the intersection of two or more algebraic surfaces. We will consider only projective spatial curves where such a curve is defined by two or more polynomial homogeneous equations:

$$
F_{i}(X, Y, Z, T)=0
$$

As in the case of planar curves, there is a natural concept of duality. The dual curve is the set of planes tangent to the curve at a simple point, that is a point $\mathbf{P}$ for which there exists $i$, such that $\nabla F_{i}(\mathbf{P}) \neq \mathbf{0}$. It turns out that the dual curve is also an algebraic variety of the dual three-dimension projective space, $\mathbb{P}^{3 *}$ (Figure 1). Moreover in general the dual curve is simply a surface of $\mathbb{P}^{3 *}$. The relation between a curve and its dual curve is bijective in the sense that a curve is completely determined by its dual curve. Therefore a spatial algebraic curve can be represented either as the solution of a family of equation or by its dual curve.

There exists a third and very useful representation of spatial algebraic curves. A line in $\mathbb{P}^{3}$ can be regarded as a point in $\mathbb{P}^{5}$ via its Plücker coordinates. If you consider the set of all lines intersecting a spatial algebraic curve, it turns out to be an algebraic variety of $\mathbb{P}^{5}$, which completely determines the original curve (Figure 2).

To obtain a more in-depth understanding of this representation of varieties, we shall first recall that the Plücker 


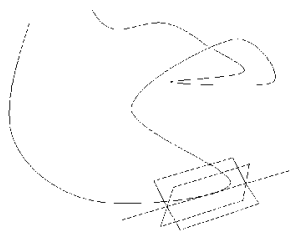

Figure 1: The dual curve is the set of planes tangent to the curve at simple points.

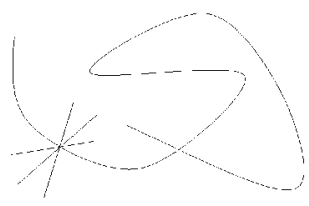

Figure 2: The set of lines intersecting a spatial curve completely determines the curve itself.

coordinates of a line satisfies a special quadratic equation, defining a quadric in $\mathbb{P}^{5}$. On the other hand every point of this quadric can be interpreted as the Plücker coordinates of a line embedded in $\mathbb{P}^{3}$. Therefore the set of lines of $\mathbb{P}^{3}$ is isomorphic (in any suitable sense) to a quadric in $\mathbb{P}^{5}$. This quadric is called the Grassmannian of lines of $\mathbb{P}^{3}$ and is denoted by $\mathbb{G}(1,3)$.

Consider next an irreducible algebraic curve embedded in $\mathbb{P}^{3}$. We have mentioned that the set of lines which intersect this curve is an algebraic variety of $\mathbb{P}^{5}$. More precisely, this variety is the intersection of the Grassmannian $\mathbb{G}(1,3)$ with a hypersurface of $\mathbb{P}^{5}$. More formally, let $\mathcal{F}$ be the family of hypersurfaces that represent the variety of lines intersecting the curve in $\mathbb{P}^{3}$. All the elements of $\mathcal{F}$ intersect in the same sub-variety over $\mathbb{G}(1,3)$. Therefore picking any element of $\mathcal{F}$ fully determines the variety of intersecting lines of the curve, which fully determines the curve itself. We can translate this fact in terms of polynomial equations. Each element of the family $\mathcal{F}$ is defined by a polynomial. All these polynomials are identical modulo the defining equation of the Grassmannian $\mathbb{G}(1,3)$. Each of these polynomials is called the Chow polynomial of the curve.

\section{Recovering the epipolar geometry from curve correspondences}

Recovering epipolar geometry from curve correspondences requires the establishment of an algebraic relation between the two image curves, involving the fundamental matrix. Hence such an algebraic relation may be regarded as an extension of Kruppa's equations. In their original form, these equations have been introduced to compute the cameraintrinsic parameters from the projection of the absolute conic onto the two image planes [31]. However it is obvious that they still hold if one replaces the absolute conic by any conic that lies on a plane that does not meet any of the camera centers. In this form they can be used to recover the epipolar geometry from conic correspondences [23, 25]. Furthermore it is possible to extend them to any planar algebraic curve [25]. Moreover a generalization for arbitrary algebraic spatial curves is possible and is a step toward the recovery of epipolar geometry from matching curves [26].

We start by the case of planar curves which is much more simple than the general case.

We shall use the following notations in this section. $X$ will denote a spatial curve, either planar or not, whereas the image curves will be denoted by $Y_{i}, i=1,2$ and defined by polynomials $f_{i}, i=1,2$. The dual image curves $Y_{i}^{*}$ are defined by the polynomials $\phi_{i}$. The camera matrices will always be denoted by $\mathbf{M}_{i}$. F, $\mathbf{e}_{1}$ and $\mathbf{e}_{2}$ are respectively the fundamental matrix, the first and the second epipole. We will also need to consider the two following mappings, that we call in the sequel the epipolar mappings, $\gamma: \mathbf{p} \rightarrow \mathbf{e}_{1} \wedge \mathbf{p}$ and $\xi: \mathbf{p} \rightarrow \mathbf{F} \mathbf{p}$. Both are defined on the first image plane; $\gamma$ associates a point to its epipolar line in the first image, while $\xi$ sends it to its epipolar line in the second image.

\subsection{The case of planar curves}

The spatial curve $X$ is assumed to be planar and might have singular points.

\subsubsection{Introductory properties}

Let $\mathbf{H}$ be the homography induced by the plane of curve in space. Given a point $\mathbf{p}$ in the first image lying on $Y_{1}$, $\mathbf{H p}$, also denoted $h(\mathbf{p})$, must lie in the second image curve $Y_{2}$. Since these curves are irreducible, there exists a simple relation between $Y_{1}, Y_{2}$ and $h$ :

$$
\exists \lambda, \forall x, y, z, f_{2}(h(x, y, z))=\lambda f_{1}(x, y, z)
$$

Now we proceed to prove some further elementary properties about two views of a planar curve. Those properties will be necessary in the sequel. We shall denote by $\epsilon_{i}$ the set of epipolar lines, in image $i$, tangent to $Y_{i}$ at regular points.

Proposition 1 The two sets $\epsilon_{1}$ and $\epsilon_{2}$ are projectively isomorphic. Furthermore the elements of $\epsilon_{1}$ and $\epsilon_{2}$ are in correspondence through the homography $\mathbf{H}$.

Proof: The line joining $\mathbf{e}_{1}$ and $\mathbf{p}$, is tangent to $Y_{1}$ at $\mathbf{p}$ if $\lambda=0$ is a double root of the equation: $f_{1}\left(\mathbf{p}+\lambda \mathbf{e}_{1}\right)=0$. 
This is equivalent to say that $\operatorname{grad}_{\mathbf{p}}\left(f_{1}\right)^{T} \mathbf{e}_{1}=0$. Moreover if $\mathbf{p}^{\prime} \cong \mathbf{H} \mathbf{p}, \operatorname{grad}_{\mathbf{p}^{\prime}}\left(f_{2}\right)^{T} \mathbf{e}_{2}=\operatorname{grad}_{\mathbf{H p}}\left(f_{2}\right)^{T} \mathbf{H e}_{1}=$ $\mathrm{d} f_{2}(h(\mathbf{p})) \circ \mathrm{d} h(\mathbf{p}) \cdot \mathbf{e}_{1}=\mathrm{d}\left(f_{2} \circ h\right)(\mathbf{p}) \cdot \mathbf{e}_{1}=\lambda \mathrm{d} f_{1}(\mathbf{p}) \cdot \mathbf{e}_{1}=$ $\lambda \operatorname{grad}_{\mathbf{p}}\left(f_{1}\right)^{T} \mathbf{e}_{1}$.

Therefore the line generated by $\mathbf{e}_{1}$ and $\mathbf{p}$ is tangent to $Y_{1}$ if and only if the line given by $\mathbf{e}_{2} \wedge \mathbf{H p}$ is tangent to $Y_{2}$. Given a line $\mathbf{l} \in \epsilon_{1}$, its corresponding line $\mathbf{l}^{\prime} \in \epsilon_{2}$ is given by: $\mathbf{H}^{-T} \mathbf{l}=\mathbf{l}^{\prime}$. ${ }^{1}$

Note that since epipolar lines are transformed in the same way through any regular homography, the two sets $\epsilon_{1}$ and $\epsilon_{2}$ are in fact projectively related by any homography.

Proposition 2 The inflexions (respectively the singularities) of the two image curves are projectively related by the homography through the plane of the curve in space.

Proof: The simple relation (1) implies this double property:

$$
\begin{gathered}
{\left[\begin{array}{c}
\frac{\partial f_{1}}{\partial x}(\mathbf{p}) \\
\frac{\partial f_{1}}{\partial y}(\mathbf{p}) \\
\frac{\partial f_{1}}{\partial y}(\mathbf{p})
\end{array}\right]=\mathbf{H}^{T}\left[\begin{array}{c}
\frac{\partial f_{2}}{\partial x}(\mathbf{H p}) \\
\frac{\partial f_{2}}{\partial y}(\mathbf{H p}) \\
\frac{\partial f_{2}}{\partial y}(\mathbf{H p})
\end{array}\right] .} \\
{\left[\frac{\partial^{2} f_{1}}{\partial x_{i} \partial x_{j}}(\mathbf{p})\right]=\mathbf{H}^{T}\left[\frac{\partial^{2} f_{2}}{\partial x_{i} \partial x_{j}}(\mathbf{H} \mathbf{p})\right] \mathbf{H}}
\end{gathered}
$$

The first relations implies the conservation of the singularities by homography, whereas the second relation implies the conservation of the whole Hessian curve by homography.

Using further derivation, we immediately get:

Proposition 3 The multiplicity and the number of distinct tangents at a singularity is the same in both images.

\subsubsection{Extended Kruppa's equations}

Roughly speaking, the extended Kruppa's equations state that the sets of epipolar lines tangent to the curve in each image are projectively related. A similar observation has been made in [2] for epipolar lines tangent to apparent contours of objects, but it was used within an optimization scheme. Here we are looking for closed-form solutions, where no initial guesses are required. In order to develop such a closed-form solution for the computation of the epipolar geometry, we need a more quantitative approach, which is given by the following theorem:

Theorem 1 For a generic position of the camera centers, the dual image curves and the epipolar mappings are related as follows. There exists a non-zero scalar $\lambda \in \mathbb{C}$, such as for all $\mathbf{p}$ in the first image plane, the following equality holds:

$$
\phi_{2}(\xi(\mathbf{p}))=\lambda \phi_{1}(\gamma(\mathbf{p}))
$$

${ }^{1}$ By duality $\mathbf{H}^{T}$ sends the lines of the second image plane into the lines of the first image plane. Here we have showed that $\mathbf{H}^{T}$ induces to one-to-one correspondence between $\epsilon_{2}$ and $\epsilon_{1}$.
Proof: First it is clear, by proposition 1, that both sides of the this equations define the same algebraic set, that is the union of the tangent lines to $Y_{1}$ passing through the first epipole $\mathbf{e}_{1}$. This set has been denoted by $\epsilon_{1}$. Moreover by propositions 2 and 3 the two dual curves have same degree, which is a necessary condition for the equation to hold. It is left to show that each tangent appears with the same multiplicity in each side. It is easily checked by a short computation: $\phi_{2}(\xi(\mathbf{p}))=\phi_{2}\left(\mathbf{e}_{2} \wedge \mathbf{H} \mathbf{p}\right)=\phi_{2}\left(h\left(\mathbf{e}_{2}\right) \wedge h(\mathbf{p})\right) \cong$ $\phi_{2} \circ\left({ }^{t} h\right)^{-1}(\mathbf{p}) .{ }^{2}$ Then it is sufficient to see that the dual formulation of equation (1) is given by $\phi_{2} \circ\left({ }^{t} h\right)^{-1} \cong \phi_{1}$.

\subsubsection{Recovering epipolar geometry from matching conics}

Let $\mathbf{C}_{1}$ (respectively $\mathbf{C}_{2}$ ) be the full rank (symmetric) matrix of the conic in the first (respectively second) image. The equations of the dual curves are $\phi_{1}(u, v, w)=\mathbf{1}^{T} \mathbf{C}_{1}^{*} \mathbf{l}=0$ and $\phi_{2}(u, v, w)=\mathbf{1}^{T} \mathbf{C}_{2}^{*} \mathbf{l}=0$ where $\mathbf{l}=[u, v, w]^{T}, \mathbf{C}_{1}^{*}$ and $\mathbf{C}_{2}^{\star}$ are the adjoint matrices of $\mathbf{C}_{1}$ and $\mathbf{C}_{2}$ (see [39]).

Hence the extended Kruppa's equations reduce in that case to the classical ones:

$$
\mathbf{F}^{T} \mathbf{C}_{2}^{*} \mathbf{F} \cong\left[\mathbf{e}_{1}\right]_{\times} \mathbf{C}_{1}^{*}\left[\mathbf{e}_{1}\right]_{\times} .
$$

From equation (3), one can extract a set, denoted $\mathcal{E}_{\lambda}$, of six equations on $\mathbf{F}, \mathbf{e}_{1}$ and an auxiliary unknown $\lambda$. By eliminating $\lambda$ it is possible to get five bi-homogeneous equations on $\mathbf{F}$ and $\mathbf{e}_{1}$.

Theorem 2 The six equations, $\mathcal{E}_{\lambda}$, are algebraically independent.

Proof: Using the following regular isomorphism: $\left(\mathbf{F}, \mathbf{e}_{1}, \lambda\right) \longmapsto\left(\mathbf{D}_{2}^{\star} \mathbf{F} \mathbf{D}_{1}^{-1}, \mathbf{D}_{1} \mathbf{e}_{1}, \lambda\right)=(\mathbf{X}, \mathbf{y}, \lambda)$, where $\mathbf{D}_{1}=\sqrt{\mathbf{C}_{1}}$ and $\mathbf{D}_{2}^{\star}=\sqrt{\mathbf{C}_{2}^{\star}}$, the original equations are mapped into the upper-triangle of $\mathbf{X}^{T} \mathbf{X}=\lambda[\mathbf{y}]_{\times}^{2}$. Given this simplified form, it is possible of compute a Gröbner basis [5]. Then we can compute the dimension of the affine variety in the variables $(\mathbf{X}, \mathbf{y}, \lambda)$, defined by these six equations. The dimension is 7 , which shows that the equations are algebraically independent.

Note that the equations $\mathcal{E}_{\lambda}$ imply that $\mathbf{F e}_{1}=\mathbf{0}$ (one can easily deduce it from the equation $3^{3}$ ). In order to count the number of matching conics, in generic positions, that are necessary and sufficient to recover the epipolar geometry, we eliminate $\lambda$ from $\mathcal{E}_{\lambda}$ and we get a set $\mathcal{E}$ that defines a variety $V$ of dimension 7 in a 12-dimensional affine space, whose points are $\left(\mathbf{e}_{1}, \mathbf{F}\right)$. The equations in $\mathcal{E}$ are bihomogeneous in $\mathbf{F}$ and $\mathbf{e}_{1}$ and $V$ can also be regarded as a

\footnotetext{
${ }^{2}$ Indeed for a regular $3 \times 3$ matrix $\mathbf{H}: \mathbf{H x} \wedge \mathbf{H y}=\operatorname{det}(H) \mathbf{H}^{-T}(\mathbf{x} \wedge$ $\mathbf{y})$. Then since $\phi_{2}$ is a homogeneous polynomial, the last equality is true up to the scale factor, $\operatorname{det}(H)^{\operatorname{deg}\left(\phi_{2}\right)}$.

${ }^{3}$ It is clear that we have: $\mathbf{F}^{T} \mathbf{C}_{2}^{\star} \mathbf{F e} 1=\mathbf{0}$. For any matrix $\mathbf{M}$, we have: $\operatorname{ker}\left(\mathbf{M}^{T}\right)=\operatorname{im}(\mathbf{M})^{T}$. In addition, $\mathbf{C}_{2}$ is invertible. Hence $\mathbf{F} \mathbf{e}_{1}=\mathbf{0}$.
} 
variety of dimension 5 into the bi-projective space $\mathbb{P}^{2} \times \mathbb{P}^{8}$, where $\left(\mathbf{e}_{1}, \mathbf{F}\right)$ lie. Now we project $V$ into $\mathbb{P}^{8}$, by eliminating $\mathbf{e}_{1}$ from the equations, we get a new variety $V_{f}$ which is still of dimension 5 and which is contained into the variety defined by $\operatorname{det}(\mathbf{F})=0$, whose dimension is $7^{4}$. Therefore two pairs of matching conics in generic positions define two varieties isomorphic to $V_{f}$ which intersect in a threedimensional variety $(5+5-7=3)$. A third conic in generic position will reduce the intersection to a one-dimensional variety $(5+3-7=1)$. A fourth conic will reduce the system to a zero-dimensional variety. These results can be compiled into the following theorem:

Theorem 3 \{Four conics\} or $\{$ three conics and a point $\}$

or $\{$ two conics and three points $\}$

or

\{one conic and five points\}

in generic positions are sufficient to compute the epipolar geometry. [23].

A similar result has been formulated independently in

\subsubsection{Recovering epipolar geometry from higher order planar curves}

Now we proceed to analysis the case of planar algebraic curves which degree $d \geq 3$. Since one cannot get a threedimensional reconstruction from a single planar algebraic curve, a further analysis of the dimension of the set of solutions of the extended Kruppa's equations, in that case, is not really relevant. Therefore we concentrate on recovering the epipolar geometry through the computation of the homography induced by the plane of the curve in space.

We will show next that a single matching pair of planar curves, which genus $g \geq 1$ (which implies that the degree $d \geq 3$ ), is sufficient for uniquely recovering the homography matrix induced by the plane of the curve in space, whereas two pairs of matching curves (residing on distinct planes) are sufficient for recovering the fundamental matrix.

From equation (1), we get $\left(\begin{array}{c}d+2 \\ d\end{array}\right)-1$ equations on the entries of the homography matrix. Let $V$ the variety in $\mathbb{P}^{8}$ defined by these equations. We give a sufficient conditions for $V$ to be discrete.

Proposition 4 For $V$ being a finite set, it is sufficient that $g \geq 1$.

Proof: The homography matrix must leave the Weierstrass points $([15,19])$ globally invariant. The number of Weierstrass point is always finite and is at least $2 g+2$. Then for $g \geq 1$, there are enough Weierstrass points to constraint the homography matrix up to a finite-fold ambiguity.

Hence follows immediately:

${ }^{4}$ Since it must be contained into the projection to $\mathbb{P}^{8}$ of the hypersurface defined by $\operatorname{det}\left(\mathbf{F e}_{1}\right)=0$
Theorem 4 Two planar algebraic curves which genus are greater or equal to 1 and that are lying on two generic planes are sufficient to recover the epipolar geometry.

However solving equations (1) requires big machinery either symbolic or numerical. Moreover the computation of Weierstrass points, introduced in the proof of the previous proposition, is quite heavy. Hence we propose a simpler algorithm that works for a large category of planar curves. This simpler algorithm is true for non-oversingular curves, e.g. when a technical condition about the singularities of the curve holds. A curve of degree $d$, whose only singular points are either nodes or cusps, satisfy the Plücker's formula (see [43]):

$$
3 d(d-2)=i+6 \delta+8 \kappa,
$$

where $i$ is the number of inflexion points, $\delta$ is the number of nodes, and $\kappa$ is the number of cusps. For our purpose, a curve is said to be non-oversingular when its only singularities are nodes and cusps and when $i+s \geq 4$, where $s$ is the number of all singular points.

Since the inflexion and singular points in both images are projectively related through the homography matrix (proposition 2), one can compute the homography through the plane of the curve in space as follows:

1. Compute the Hessian curves in both images.

2. Compute the intersection of the curve with its Hessian in both images. The output is the set of inflexion and singular points.

3. Discriminate between inflexion and singular points by the additional constraint for each singular point a: $\operatorname{grad}_{\mathbf{a}}(f)=\mathbf{0}$.

At first sight, there are $i ! \times s !$ possible correspondences between the sets of inflexion and singular points in the two images. But it is possible to further reduce the combinatory by separating the points into two categories. The points are normalized such that the last coordinates is 1 or 0 . Then separate real points from complex points. It is clear that real points are mapped to real points. Now by genericity (the plane of the curve in space does not pass through the camera centers), complex points are mapped to complex points. Indeed consider the point $\mathbf{p}=(\alpha, \beta, \epsilon)+i(\gamma, \delta, 0)$, where $\epsilon \in\{0,1\}$. Let $\mathbf{A}=\left(a_{i j}\right)_{i j}$ be the homography matrix. Then $\mathbf{A p}=\left(a_{11}(\alpha+i \gamma)+a_{12}(\beta+i \delta)+a_{13} \epsilon, a_{21}(\alpha+\right.$ $\left.i \gamma)+a_{22}(\beta+i \delta)+a_{23} \epsilon, a_{31}(\alpha+i \gamma)+a_{32}(\beta+i \delta)+a_{33} \epsilon\right)$. In order Ap to be a real point, two algebraic conditions on the $\mathbf{A}$ and $\mathbf{p}$ must be satisfied. This cannot hold in general. Therefore we can conclude that each category of the first image must be matched with the same category in the second image. This should be used to reduce the combinatory. Then the right solution can be selected as it should be the 
one that makes the system of equations extracted from (1) the closest to zero or the one that minimizes the Hausdorff distance (see [22]) between the set of points from the second image curve and the reprojection of the set of points from the first image curve into the second image. For better results, one can compute the Hausdorff distance on inflexion and singular points separately, within each category.

\subsection{The case of non-planar}

Here $X$ be a smooth irreducible curve in $\mathbb{P}^{3}$, whose degree is $d \geq 2$. Since the case of planar curve has been treated above, $X$ is assumed to be a non-planar curve. Before defining and proving the extended Kruppa's equations for arbitrary curve, we need to establish a number of facts about the projection of a spatial curve onto a plane by a pinhole camera.

\subsubsection{Single view of a spatial curve}

Let $\mathbf{M}$ be the camera matrix, $\mathbf{O}$ the camera center, $\mathcal{R}$ the retinal plane and $Y$ the image curve. Our first concern is the study of the singularities of the $Y$.

Proposition 5 The curve $Y$ will always contain singularities.

Proof: Singularities correspond to optical rays that meet the curve $X$ in at least two distinct points or are tangent to $\mathrm{X}$. Let $\mathbb{G}(1,3)$ denote the Grassmannian of lines in $\mathbb{P}^{3}$. Consider the subvariety $S_{X}$ of $\mathbb{G}(1,3)$ generated by the set of chords and tangents of $X$, a chord being a line meeting $X$ at least twice. Let $\mathcal{S}(X)=\cup_{L \in S_{X}} L$ be the union of lines that are elements of $S_{X}$. It is well known that $\mathcal{S}(X)$ is an irreducible subvariety of $\mathbb{P}^{3}$ and that $\operatorname{dim}(\mathcal{S}(X))=3$, unless $X$ is planar, which has been excluded (see [18]). Hence $\mathcal{S}(X)=\mathbb{P}^{3}$ and $\mathbf{O} \in \mathcal{S}(X)$, that is, at least one chord is passing through $\mathbf{O}$.

The process of singularity formation with projection is illustrated in the figure 3 . In the proposition below we will investigate the nature of those singularities.

Proposition 6 For a generic position of the camera center, the only singularities of $Y$ will be nodes.

Proof: This is a well known result. See [21] for further details.

We define the class of the planar curve to be the degree of its dual curve. Let $m$ be the class of $Y$. We prove that $m$ is constant for a generic position of the camera center.

Proposition 7 For a generic position of $\mathbf{O}$, the class of $Y$ is constant.

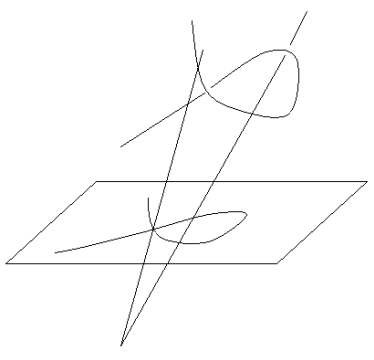

Figure 3: A singularity of the image curve corresponds to an optical ray meeting the curve in space at least twice.

Proof: For each position of $\mathbf{O} \notin \mathcal{R}$, we get a particular curve $Y$ or equivalently $Y^{\star}$, which is the dual curve of $Y$. Let $\mathcal{Y}$ be this family of dual curves parameterized by $\mathbf{O}$. Then $\mathcal{Y} \subset \mathbb{P}^{2 \star} \times \mathbb{P}^{3}$. Then it is known that $\mathcal{Y}$ is flat $[21$, $18,8,7]$ over an open set of $\mathbb{P}^{3}$. Since $\mathbb{P}^{3}$ is irreducible, this open set must be dense. On the other hand, the degree is constant for a flat family of varieties. Hence the degree of $Y^{\star}$ is constant for a generic position of $\mathbf{O}$.

Note that using Proposition 6, it is possible to give a formula for $m$ as a function of the degree $d$ and the genus $g$ of $X$. For a generic position of $\mathbf{O}, Y$ will have same degree and genus as $X$. As mentionned above, we have:

$$
\begin{gathered}
m=d(d-1)-2(\sharp \text { nodes }), \\
g=\frac{(d-1)(d-2)}{2}-(\sharp \text { nodes }),
\end{gathered}
$$

where $\sharp$ nodes denotes the number of nodes of $Y$ (note that this includes complex nodes). Hence the genus, the degree and the class are related by:

$$
m=2 d+2 g-2 \text {. }
$$

\subsubsection{Extended Kruppa's equations}

We are ready now to investigate the recovery of the epipolar geometry from matching curves. The major result here is that the Extended Kruppa's Equations still hold in the general case of non-planar algebraic curves.

\section{Theorem 5 Extended Kruppa's equations}

For a generic position of the camera centers with respect to the curve in space, there exists a non-zero scalar $\lambda$, such that for all points $\mathbf{p}$ in the first image, the following equality holds:

$$
\phi_{2}(\xi(\mathbf{p}))=\lambda \phi_{1}(\gamma(\mathbf{p}))
$$


Once again observe that if $X$ is a conic and $\mathbf{C}_{1}$ and $\mathbf{C}_{2}$ the matrices that respectively represent $Y_{1}$ and $Y_{2}$, the extended Kruppa's equations reduce to the classical Kruppa's equations, that is: $\left[\mathbf{e}_{1}\right]_{x}^{T} \mathbf{C}_{1}^{\star}\left[\mathbf{e}_{1}\right]_{x} \cong \mathbf{F}^{T} \mathbf{C}_{2}^{\star} \mathbf{F}$, where $\mathbf{C}_{1}^{\star}$ and $\mathbf{C}_{2}^{\star}$ are the adjoint matrices of $\mathbf{C}_{1}$ and $\mathbf{C}_{2}$.

Proving these extended Kruppa's equations requires the establishment of these three steps:

1. $\phi_{1}$ and $\phi_{2}$ have equal degrees, that is, the image curves have the same class.

2. If $\epsilon_{i}$ is the set of epipolar lines tangent to the curve in image $i$, then $\epsilon_{1}$ and $\epsilon_{2}$ must be projectively isomorphic.

3. Moreover in each corresponding pair of epipolar lines, $\left(\mathbf{l}, \mathbf{l}^{\prime}\right) \in \epsilon_{1} \times \epsilon_{2}$, the multiplicity of $\mathbf{l}$ and $\mathbf{l}^{\prime}$ as points of the dual curves $Y_{1}^{\star}$ and $Y_{2}^{\star}$ must be the same.

The first step is obviously necessary for the equation (4) to hold. It is true, as stated in Proposition 7. As previously, let $m$ be the common degree of $\phi_{1}$ and $\phi_{2}$. The second point is necessary to prove that both sides of equation (4) represent the same geometric set, that is here the union of lines in $\epsilon_{1}$. This point holds thanks to the following lemma:

Lemma 1 The sets of epipolar lines tangent to the image curve in each image are projectively related.

Proof: This is due to the fact that a pair of corresponding epipolar lines is the trace in the images of a plane containing the baseline joining the two camera centers. Hence the two lines are tangent to the image curves if and only if the plane they define (which contains the baseline) is tangent to the curve in space. Therefore the two sets $\epsilon_{1}$ and $\epsilon_{2}$ are composed of the traces in the images of the planes containing the baseline and tangent to the curve in space. Hence these two sets are projectively related.

Finally we can prove Theorem 5.

Proof: Since each side of equation (4) represents the union of lines in $\epsilon_{1}$, it can be factorized into linear factors, satisfying the following:

$$
\begin{gathered}
\phi_{1}(\gamma(x, y, z))=\prod_{i}\left(\alpha_{1 i} x+\alpha_{2 i} y+\alpha_{3 i} z\right)^{a_{i}} \\
\phi_{2}(\xi(x, y, z))=\prod_{i} \lambda_{i}\left(\alpha_{1 i} x+\alpha_{2 i} y+\alpha_{3 i} z\right)^{b_{i}}
\end{gathered}
$$

where $\sum_{i} a_{i}=\sum_{j} b_{j}=m$. It is left to prove that for all $i$, $a_{i}=b_{i}$. Observe that $a_{i}>1$, when the epipolar line is tangent to the image curve in at least two distinct points. Hence one must prove that the number of tangency points for each element of $\epsilon_{1}$ is the same for its corresponding element in $\epsilon_{2}$. This number must also be the number of tangency points of the plane, defined by these two corresponding elements in $\epsilon_{1}$ and $\epsilon_{2}$. Hence this third assertion holds using the same argument as in Lemma 1.

By eliminating the scalar $\lambda$ from the extended Kruppa's equations (4) we obtain a set of bi-homogeneous equations in $\mathbf{F}$ and $\mathbf{e}_{1}$. Hence they define a variety in $\mathbb{P}^{2} \times \mathbb{P}^{8}$. This gives rise to an important question. How many of those equations are algebraically independent, or in other words what is the dimension of the set of solutions? This is the issue of the next section.

\subsubsection{Dimension of the set of solutions}

Let $\left\{E_{i}\left(\mathbf{F}, \mathbf{e}_{1}\right)\right\}_{i}$ be the set of bi-homogeneous equations on $\mathbf{F}$ and $\mathbf{e}_{1}$, extracted from the extended Kruppa's equations (4). Our first concern is to determine whether all solutions of equation (4) are admissible, that is whether they satisfy the usual constraint $\mathbf{F} \mathbf{e}_{1}=0$. Indeed we prove the following statement:

Proposition 8 As long as there are at least 2 distinct lines through $\mathbf{e}_{1}$ tangent to $Y_{1}$, equation (4) implies that $\operatorname{rank} \mathbf{F}=$ 2 and $\mathbf{F} \mathbf{e}_{1}=\mathbf{0}$.

Proof: The variety defined by $\phi_{1}(\gamma(\mathbf{p}))$ is then a union of at least 2 distinct lines through $\mathbf{e}_{1}$. If equation (4) holds, $\phi_{2}(\xi(\mathbf{p}))$ must define the same variety.

There are 2 cases to exclude: If $\operatorname{rankF}=3$, then the curve defined by $\phi_{2}(\xi(\mathbf{p}))$ is projectively equivalent to the curve defined by $\phi_{2}$, which is $Y_{1}^{\star}$. In particular, it is irreducible.

If $\operatorname{rank} \mathbf{F}<2$ or $\operatorname{rank} \mathbf{F}=2$ and $\mathbf{F} \mathbf{e}_{1} \neq \mathbf{0}$, then there is some $\mathbf{a}$, not a multiple of $\mathbf{e}_{1}$, such that $\mathbf{F a}=\mathbf{0}$. Then the variety defined by $\phi_{2}(\xi(\mathbf{p}))$ is a union of lines through a. In neither case can this variety contain two distinct lines through $\mathbf{e}_{1}$, so we must have $\operatorname{rank} \mathbf{F}=2$ and $\mathbf{F} \mathbf{e}_{1}=\mathbf{0}$.

As a result, in a generic situation every solution of $\left\{E_{i}\left(\mathbf{F}, \mathbf{e}_{1}\right)\right\}_{i}$ is admissible. Let $V$ be the subvariety of $\mathbb{P}^{2} \times \mathbb{P}^{8} \times \mathbb{P}^{2}$ defined by the equations $\left\{E_{i}\left(\mathbf{F}, \mathbf{e}_{1}\right)\right\}_{i}$ together with $\mathbf{F} \mathbf{e}_{1}=\mathbf{0}$ and $\mathbf{e}_{2}{ }^{T} \mathbf{F}=\mathbf{0}^{T}$, where $\mathbf{e}_{2}$ is the second epipole. We next compute the lower bound on the dimension of $V$, after which we would be ready for the calculation itself.

Proposition 9 If $V$ is non-empty, the dimension of $V$ is at least $7-m$.

Proof: Choose any line $\mathbf{l}$ in $\mathbb{P}^{2}$ and restrict $\mathbf{e}_{1}$ to the affine piece $\mathbb{P}^{2} \backslash 1$. Let $(x, y)$ be homogeneous coordinates on 1 . If $\mathbf{F e}_{1}=\mathbf{0}$, the two sides of equation (4) are both unchanged by replacing $\mathbf{p}$ by $\mathbf{p}+\alpha \mathbf{e}_{1}$. So equation (4) will hold for all $\mathbf{p}$ if it holds for all $\mathbf{p} \in \mathbf{1}$. Therefore equation (4) is equivalent to the equality of 2 homogeneous polynomials of degree $m$ in $x$ and $y$, which in turn is equivalent to the equality of $(m+1)$ coefficients. After eliminating $\lambda$, we 
have $m$ algebraic conditions on $\left(\mathbf{e}_{1}, \mathbf{F}, \mathbf{e}_{2}\right)$ in addition to $\mathbf{F e}_{1}=\mathbf{0}, \mathbf{e}_{2}{ }^{T} \mathbf{F}=\mathbf{0}^{T}$.

The space of all epipolar geometries, that is, solutions to $\mathbf{F e}_{1}=\mathbf{0}, \mathbf{e}_{2}{ }^{T} \mathbf{F}=\mathbf{0}^{T}$, is irreducible of dimension 7 . Therefore, $V$ is at least $(7-m)$-dimensional.

For the calculation of the dimension of $V$ we introduce some additional notations. Given a triplet $\left(\mathbf{e}_{1}, \mathbf{F}, \mathbf{e}_{2}\right) \in$ $\mathbb{P}^{2} \times \mathbb{P}^{8} \times \mathbb{P}^{2}$, let $\left\{\mathbf{q}_{1 \alpha}\left(\mathbf{e}_{1}\right)\right\}$ (respectively $\left\{\mathbf{q}_{2 \alpha}\left(\mathbf{e}_{2}\right)\right\}$ ) be the tangency points of the epipolar lines through $\mathbf{e}_{1}$ (respectively $\mathbf{e}_{2}$ ) to the first (respectively second) image curve. Let $\mathbf{Q}_{\alpha}\left(\mathbf{e}_{1}, \mathbf{e}_{2}\right)$ be the $3 \mathrm{~d}$ points projected onto $\left\{\mathbf{q}_{1 \alpha}\left(\mathbf{e}_{1}\right)\right\}$ and $\left\{\mathbf{q}_{2 \alpha}\left(\mathbf{e}_{2}\right)\right\}$. Let $\mathbf{L}$ be the baseline joining the two camera centers. We next provide a sufficient condition for $V$ to be discrete.

Proposition 10 For a generic position of the camera centers, the variety $V$ will be discrete if, for any point $\left(\mathbf{e}_{1}, \mathbf{F}, \mathbf{e}_{2}\right) \in V$, the union of $\mathbf{L}$ and the points $\mathbf{Q}_{\alpha}\left(\mathbf{e}_{1}, \mathbf{e}_{2}\right)$ is not contained in any quadric surface.

Proof: For generic camera positions, there will be $m$ distinct points $\left\{\mathbf{q}_{1 \alpha}\left(\mathbf{e}_{1}\right)\right\}$ and $\left\{\mathbf{q}_{2 \alpha}\left(\mathbf{e}_{2}\right)\right\}$, and we can regard $\mathbf{q}_{1 \alpha}, \mathbf{q}_{2 \alpha}$ locally as smooth functions of $\mathbf{e}_{1}, \mathbf{e}_{2}$.

We let $W$ be the affine variety in $\mathbb{C}^{3} \times \mathbb{C}^{9} \times \mathbb{C}^{3}$ defined by the same equations as $V$. Let $\Theta=\left(\mathbf{e}_{1}, \mathbf{F}, \mathbf{e}_{2}\right)$ be a point of $W$ corresponding to a non-isolated point of $V$. Then there is a tangent vector $\vartheta=\left(\mathbf{v}, \Phi, \mathbf{v}^{\prime}\right)$ to $W$ at $\Theta$ with $\Phi$ not a multiple of $\mathbf{F}$.

If $\chi$ is a function on $W, \nabla_{\Theta, \vartheta}(\chi)$ will denote the derivative of $\chi$ in the direction defined by $\vartheta$ at $\Theta$. For

$$
\chi_{\alpha}\left(\mathbf{e}_{1}, \mathbf{F}, \mathbf{e}_{2}\right)=\mathbf{q}_{2 \alpha}\left(\mathbf{e}_{2}\right)^{T} \mathbf{F} \mathbf{q}_{1 \alpha}\left(\mathbf{e}_{1}\right),
$$

the extended Kruppa's equations imply that $\chi_{\alpha}$ vanishes identically on $W$, so its derivative must also vanish. This yields

$$
\begin{aligned}
\nabla_{\Theta, \vartheta}\left(\chi_{\alpha}\right)= & \left(\nabla_{\Theta, \vartheta}\left(\mathbf{q}_{2 \alpha}\right)\right)^{T} \mathbf{F} \mathbf{q}_{1 \alpha} \\
& +\mathbf{q}_{2 \alpha}^{T} \Phi \mathbf{q}_{1 \alpha}+\mathbf{q}_{2 \alpha}^{T} \mathbf{F}\left(\nabla_{\Theta, \vartheta}\left(\mathbf{q}_{1 \alpha}\right)\right) \\
= & 0 .
\end{aligned}
$$

We shall prove that $\nabla_{\Theta, \vartheta}\left(\mathbf{q}_{1 \alpha}\right)$ is in the linear span of $\mathbf{q}_{1 \alpha}$ and $\mathbf{e}_{1}$. (This means that when the epipole moves slightly, $\mathbf{q}_{1 \alpha}$ moves along the epipolar line, see figure 4.)

Consider $\kappa(t)=f\left(\mathbf{q}_{1 \alpha}\left(\mathbf{e}_{1}+t \mathbf{v}\right)\right)$, where $f$ is the polynomial defining the image curve $Y_{1}$. Since $\mathbf{q}_{1 \alpha}\left(\mathbf{e}_{1}+t \mathbf{v}\right) \in$ $Y_{1}, \kappa \equiv 0$, so the derivative $\kappa^{\prime}(0)=0$. On the other hand, $\kappa^{\prime}(0)=\nabla_{\Theta, \vartheta}\left(f\left(\mathbf{q}_{1 \alpha}\right)\right)=\operatorname{grad}_{\mathbf{q}_{1 \alpha}}(f)^{T} \nabla_{\Theta, \vartheta}\left(\mathbf{q}_{1 \alpha}\right)$.

Thus we have $\operatorname{grad}_{\mathbf{q}_{1 \alpha}}(f)^{T} \nabla_{\Theta, \vartheta}\left(\mathbf{q}_{1 \alpha}\right)=0$. But also $\operatorname{grad}_{\mathbf{q}_{1 \alpha}}(f)^{T} \mathbf{q}_{1 \alpha}=0$ and $\operatorname{grad}_{\mathbf{q}_{1 \alpha}}(f)^{T} \mathbf{e}_{1}=0$. Since $\operatorname{grad}_{\mathbf{q}_{1 \alpha}}(f) \neq \mathbf{O}\left(\mathbf{q}_{1 \alpha}\right.$ is not a singular point of the curve), this shows that $\nabla_{\Theta, \vartheta}\left(\mathbf{q}_{1 \alpha}\right), \mathbf{q}_{1 \alpha}$, and $\mathbf{e}_{1}$ are linearly dependent. $\mathbf{q}_{1 \alpha}$ and $\mathbf{e}_{1}$ are linearly independent, so $\nabla_{\Theta, \vartheta}\left(\mathbf{q}_{1 \alpha}\right)$ must be in their linear span.

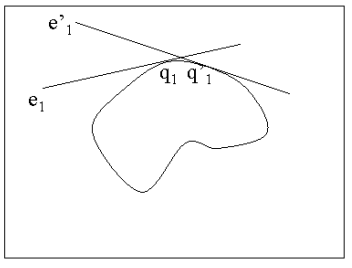

Figure 4: The point $\mathbf{q}_{1 \alpha}\left(\mathbf{e}_{1}\right)$ is a smooth function of $\mathbf{e}_{1}$. When $\mathbf{e}_{1}$ moves slightly, $\mathbf{q}_{1 \alpha}\left(\mathbf{e}_{1}\right)$ moves along the epipolar line.

We have that $\mathbf{q}_{2 \alpha}^{T} \mathbf{F} \mathbf{e}_{1}=\mathbf{q}_{2 \alpha}^{T} \mathbf{F} \mathbf{q}_{1 \alpha}=0$, so $\mathbf{q}_{2 \alpha}^{T} \mathbf{F} \nabla_{\Theta, \vartheta}\left(\mathbf{q}_{1 \alpha}\right)=0$ : the third term of equation (5) vanishes. In a similar way, the first term of equation (5) vanishes, leaving

$$
\mathbf{q}_{2 \alpha}^{T} \Phi \mathbf{q}_{1 \alpha}=0 \text {. }
$$

The derivative of $\chi\left(\mathbf{e}_{1}, \mathbf{F}, \mathbf{e}_{2}\right)=\mathbf{F e}_{1}$ must also vanish, which yields:

$$
\mathbf{e}_{2}^{T} \Phi \mathbf{e}_{1}=0 .
$$

From the first equality, we deduce that for every $\mathbf{Q}_{\alpha}$, we have:

$$
\mathbf{Q}_{\alpha}^{T} \mathbf{M}_{2}^{T} \Phi \mathbf{M}_{1} \mathbf{Q}_{\alpha}=0 .
$$

From the second equality, we deduce that every point $\mathbf{P}$ lying on the baseline must satisfy:

$$
\mathbf{P}^{T} \mathbf{M}_{2}^{T} \Phi \mathbf{M}_{1} \mathbf{P}=0
$$

The fact that $\Phi$ is not a multiple of $F$ implies that $\mathbf{M}_{2}^{T} \Phi \mathbf{M}_{1} \neq 0$, so together these two last equations mean that the union $\mathbf{L} \cup\left\{\mathbf{Q}_{\alpha}\right\}$ lies on a quadric surface. Thus if there is no such quadric surface, every point in $V$ must be isolated.

Observe that this result is consistent with the previous proposition, since there always exist a quadric surface containing a given line and six given points. However in general there is no quadric containing a given line and seven given points. Therefore we can conclude with the following theorem.

Theorem 6 For a generic position of the camera centers, the extended Kruppa's equations define the epipolar geometry up to a finite-fold ambiguity if and only if $m \geq 7$.

Since different curves in generic position give rise to independent equations, this result means that the sum of the classes of the image curves must be at least 7 for $V$ to be a finite set. Observe that this result is consistent with the fact 
that four conics ( $m=2$ for each conic) in general position are sufficient to compute the fundamental matrix, as shown in $[23,25]$. Now we proceed to translate the result in terms of the geometric properties of $X$ directly using the degree and the genus of $X$, related to $m$ by the following relation: $m=2 d+2 g-2$. Here are some examples for sets of curves that allow the recovery of the fundamental matrix:

1. Four conics $(d=2, g=0)$ in general position.

2. Two rational cubics $(d=3, g=0)$ in general position.

3. A rational cubic and two conics in general position.

4. Two elliptic cubics $(d=3, g=1)$ in general position (see also [25]).

5. A general rational quartic $(d=4, g=0)$, and a general elliptic quartic $(d=4, g=1)$.

\section{3D Reconstruction}

We turn our attention to the problem of reconstructing an algebraic curve from two or more views, given known camera matrices (epipolar geometries are known). The basic idea is to intersect together the cones defined by the camera centers and the image curves. However this intersection can be computed in three different spaces, giving rise to different algorithms and applications. Given the representation in one of those spaces, it is possible to compute the two other representations [24].

We shall mention that in [14] a scheme is proposed to reconstruct an algebraic curve from a single view by blowingup the projection. This approach results in a spatial curve defined up to an unknown projective transformation. In fact the only computation this reconstruction allows is the recovery of the projective properties of the curve. Moreover this reconstruction is valid for irreducible curves only. However reconstructing from two projections not only gives the projective properties of the curve, but also the relative depth of it with respect to others objects in the scene and furthermore the relative position between irreducible components.

\subsection{Homography recovery for planar curve}

We begin by restricting our attention to planar curves. We proceed to the recovery of the homography matrix induced by the plane of the curve in space. This approach reduces the reconstruction problem to finding the roots of a univariate polynomial. Let $\mathbf{e}_{i}, i=1,2$ be the first and second epipole. Let $\mathbf{S}$ be any homography, which can be extracted from the epipolar geometry by $\mathbf{S}=\left[\mathbf{e}_{2}\right]_{\times} \mathbf{F}$, where $\left[\mathbf{e}_{2}\right]_{\times}$is the matrix representing the cross-product by $\mathbf{e}_{2}$. Then the homography through the plane of the curve in space can be written as: $\mathbf{H}=\mathbf{S}+\mathbf{e}_{2} \mathbf{h}^{T}$ (see [40, 28]). We define $\mu$ to be such that: $\mathbf{H} \mathbf{e}_{1}=\mu \mathbf{e}_{2} .{ }^{5}$ As seen in equation (1), the two image curves and the homography $\mathbf{H}$ are related as follows:

$$
\exists \lambda, \forall \mathbf{p}, f_{1}(\mathbf{p})=\lambda f_{2}(\mathbf{H} \mathbf{p})
$$

This implies:

$$
\lambda \operatorname{grad}_{\mathbf{p}}\left(f_{1}\right)=\mathbf{H}^{T} \operatorname{grad}_{\mathbf{H p}}\left(f_{2}\right),
$$

for any $\mathbf{p}$ in the first image. Let $\mathbf{g}_{i}=\operatorname{grad}_{\mathbf{e}_{i}}\left(f_{i}\right), i=1,2$. Thus the previous expression, applied to $\mathbf{e}_{1}$, can be rewritten as follows:

$$
\lambda \mathbf{g}_{1}=\mu^{d-1}\left(\mathbf{S}^{T}+\mathbf{h} \mathbf{e}_{2}^{T}\right) \mathbf{g}_{2} .
$$

We define $\beta$ to be $\beta=\mathbf{e}_{2}^{T} \mathbf{g}_{2}=d f_{2}\left(\mathbf{e}_{2}\right)$ and $\eta$ to be $\eta=\frac{\lambda}{\mu^{d-1}}$. Hence $\mathbf{h}=\frac{1}{\beta}\left(\eta \mathbf{g}_{1}-\mathbf{S}^{T} \mathbf{g}_{2}\right)$. Thus $\mathbf{H}=$ $\mathbf{S}+\frac{1}{\beta} \mathbf{e}_{2}\left(\eta \mathbf{g}_{1}^{T}-\mathbf{g}_{2} \mathbf{S}\right)=\left(\mathbf{I}-\frac{1}{\beta} \mathbf{e}_{2} \mathbf{g}_{2}^{T}\right) \mathbf{S}+\frac{\eta}{\beta} \mathbf{e}_{2} \mathbf{g}_{1}^{T}$. Substituting this expression of $\mathbf{H}$ into equation (1) yields:

$$
\lambda f_{1}(\mathbf{p})=f_{2}\left(\left(\mathbf{I}+\frac{1}{\beta} \mathbf{e}_{2} \mathbf{g}_{2}^{T}\right) \mathbf{S} \mathbf{p}+\frac{\eta}{\beta} \mathbf{e}_{2} \mathbf{g}_{1}^{T} \mathbf{p}\right) .
$$

Then after elimination of $\lambda$, we get a set of $\left(\begin{array}{c}d+2 \\ d\end{array}\right)-1$ equations of degree $d$ in $\eta$. Hence the problem is equivalent to find the common solutions to this set of equations. This can be achieved by picking one equation, solving it and keeping only solutions that are solutions of the whole system.

Now we turn back our attention to the general case. We shall investigate three different types of representation, each leading to a particular reconstruction algorithm.

\subsection{Reconstruction in Point Space}

\subsubsection{The general case}

Let the camera projection matrices be $[\mathbf{I} ; \mathbf{0}]$ and $\left[\mathbf{S} ; \mathbf{e}_{2}\right]$, where $\mathbf{S}=-\frac{\left[\mathbf{e}_{2}\right]_{\times}}{\left\|\mathbf{e}_{2}\right\|^{2}} \mathbf{F}$, see [28]. Hence the two cones defined by the image curves and the camera centers are given by: $\Delta_{1}(\mathbf{P})=f_{1}([\mathbf{I} ; \mathbf{0}] \mathbf{P})$ and $\Delta_{2}(\mathbf{P})=f_{2}\left(\left[\mathbf{S} ; \mathbf{e}_{2}\right] \mathbf{P}\right)$. The reconstruction is defined as the curve whose equations are $\Delta_{1}=0$ and $\Delta_{2}=0$. It is clear that the original space curves is contained within the intersection on these two viewing cones. However since each cone has degree $d$ (the same than the space curve), by Bezout theorem, the intersection must have degree $d^{2}$. This implies that the intersection contains more than just the original space curve. It turns out, by the following theorem, that this intersection contains, in general, only two irreducible components (separated curves). One has degree $d$ and the other has degree $d(d-1)$. Therefore if $d \geq 3$, since the original space has

\footnotetext{
${ }^{5}$ Note that it is not possible to normalize $\mathbf{H}$ such that $\mathbf{H e}_{1}=\mathbf{e}_{2}$, because $\mathbf{H}$ is given as a function of $\mathbf{S}$ by $\mathbf{H}=\mathbf{S}+\mathbf{e}_{2} \mathbf{h}^{T}$, which constraints its norm.
} 
degree $d$, we can extract the right component which is the answer of the reconstruction problem. When $d=2$ (the curve is a conic), however, the reconstruction problem admits two solutions, which are both conics, and a third view is necessary to select the right conic.

Theorem 7 For a generic position of the camera centers, that is when no epipolar plane is tangent twice to the curve $X$, the curve defined by $\left\{\Delta_{1}=0, \Delta_{2}=0\right\}$ has two irreducible components. One has degree $d$ and is the actual solution of the reconstruction. The other one has degree $d(d-1)$.

Proof: For a line $1 \subset \mathbb{P}^{3}$, we write $\sigma(\mathbf{l})$ for the pencil of planes containing $\mathbf{1}$. For a point $\mathbf{p} \in \mathbb{P}^{2}$, we write $\sigma(\mathbf{p})$ for the pencil of lines through $\mathbf{p}$. There is a natural isomorphism between $\sigma\left(\mathbf{e}_{i}\right)$, the epipolar lines in image $i$, and $\sigma(\mathbf{L})$, the planes containing both camera centers. Consider the following covers of $\mathbb{P}^{1}$ :

1. $X \stackrel{\eta}{\longrightarrow} \sigma(\mathbf{L}) \cong \mathbb{P}^{1}$, taking a point $x \in X$ to the epipolar plane that it defines with the camera centers.

2. $Y_{1} \stackrel{\eta_{1}}{\longrightarrow} \sigma\left(\mathbf{e}_{1}\right) \cong \sigma(\mathbf{L}) \cong \mathbb{P}^{1}$, taking a point $y \in Y_{1}$ to its epipolar line in the first image.

3. $Y_{2} \stackrel{\eta_{2}}{\longrightarrow} \sigma\left(\mathbf{e}_{2}\right) \cong \sigma(\mathbf{L}) \cong \mathbb{P}^{1}$, taking a point $y \in Y_{2}$ to its epipolar line in the second image.

If $\rho_{i}$ is the projection $X \rightarrow Y_{i}$, then $\eta=\eta_{i} \rho_{i}$. Let $\mathcal{B}$ the union set of branch points of $\eta_{1}$ and $\eta_{2}$. It is clear that the branch points of $\eta$ are included in $\mathcal{B}$. Let $S=\mathbb{P}^{1} \backslash \mathcal{B}$, pick $t \in S$, and write $X_{S}=\eta^{-1}(S), X_{t}=\eta^{-1}(t)$. Let $\mu_{X_{S}}$ be the monodromy: $\pi_{1}(S, t) \longrightarrow \operatorname{Perm}\left(X_{t}\right)$, where $\operatorname{Perm}(Z)$ is the group of permutations of a finite set $Z$. It is well known that the path-connected components of $X$ are in one-to-one correspondence with the orbits of the action of $\operatorname{im}\left(\mu_{X_{S}}\right)$ on $X_{t}$. Since $X$ is assumed to be irreducible, it has only one component and $\operatorname{im}\left(\mu_{X_{S}}\right)$ acts transitively on $X_{t}$. Then if $\operatorname{im}\left(\mu_{X_{S}}\right)$ is generated by transpositions, this will imply that $\operatorname{im}\left(\mu_{X_{S}}\right)=\operatorname{Perm}\left(X_{t}\right)$. In order to show that $\operatorname{im}\left(\mu_{X_{S}}\right)$ is actually generated by transpositions, consider a loop in $\mathbb{P}^{1}$ centered at $t$, say $l_{t}$. If $l_{t}$ does not go round any branch point, then $l_{t}$ is homotopic to the constant path in $S$ and then $\mu_{X_{S}}\left(\left[l_{t}\right]\right)=1$. Now in $\mathcal{B}$, there are three types of branch points:

1. branch points that come from nodes of $Y_{1}$ : these are not branch points of $\eta$,

2. branch points that come from nodes of $Y_{2}$ : these are not branch points of $\eta$,

3. branch points that come from epipolar lines tangent either to $Y_{1}$ or to $Y_{2}$ : these are genuine branch points of $\eta$.
If the loop $l_{t}$ goes round a point of the first two types, then it is still true that $\mu_{X_{S}}\left(\left[l_{t}\right]\right)=1$. Now suppose that $l_{t}$ goes round a genuine branch point of $\eta$, say $b$ (and goes round no other points in $\mathcal{B}$ ). By genericity, $b$ is a simple twofold branch point, hence $\mu_{X_{S}}\left(\left[l_{t}\right]\right)$ is a transposition. This shows that $\operatorname{im}\left(\mu_{X_{S}}\right)$ is actually generated by transpositions and so $\operatorname{im}\left(\mu_{X_{S}}\right)=\operatorname{Perm}\left(X_{t}\right)$.

Now consider $\tilde{X}$, the curve defined by $\left\{\Delta_{1}=0, \Delta_{2}=\right.$ $0\}$. By Bezout's Theorem $\tilde{X}$ has degree $d^{2}$. Let $\tilde{x} \in \tilde{X}$. It is projected onto a point $y_{i}$ in $Y_{i}$, such that $\eta_{1}\left(y_{1}\right)=\eta_{2}\left(y_{2}\right)$. Hence $\tilde{X} \cong Y_{1} \times_{\mathbb{P}^{1}} Y_{2}$; restricting to the inverse image of the set $S$, we have $\tilde{X}_{S} \cong X_{S} \times_{S} X_{S}$. We can therefore identify $\tilde{X}_{t}$ with $X_{t} \times X_{t}$. The monodromy $\mu_{\tilde{X}_{S}}$ can then be given by $\mu_{\tilde{X}_{S}}(x, y)=\left(\mu_{X_{S}}(x), \mu_{X_{S}}(y)\right)$. Since $\operatorname{im}\left(\mu_{X_{S}}\right)=\operatorname{Perm}\left(X_{t}\right)$, the action of $\operatorname{im}\left(\mu_{\tilde{X}_{S}}\right)$ on $X_{t} \times X_{t}$ has two orbits, namely $\{(x, x)\} \cong X_{t}$ and $\{(x, y) \mid x \neq y\}$. Hence $\tilde{X}$ has two irreducible components. One has degree $d$ and is $X$, the other has degree $d^{2}-d=d(d-1)$.

Solving the system defined by $\Delta_{1}(\mathbf{P})=0$ and $\Delta_{2}(\mathbf{P})=$ 0 can be done by Gröbner basis computation. Then as mentioned above, for $d \geq 3$, the actual solution can be extracted. However the case of planar curves can be treated more easily.

\subsubsection{The case of planar curves}

An explicit elimination can be done to get a system on the plane parameters only, which can be useful to refine the solution of the homography found in section 4.1. Let $\mathbf{H}$ be the plane of curve in space, defined by the equation $h(\mathbf{p})=0$.

Theorem 8 The plane equation $h(\mathbf{P})=0$ satisfies the following constraint. There exists a scalar $k$ and a polynomial $r$, such that:

$$
r \times h=\Delta_{1}+k \Delta_{2} .
$$

Proof: $\Delta_{1}$ and $\Delta_{2}$ can be regarded as regular functions on the plane. Since they are irreducible polynomials and vanish on the plane on the same irreducible curve and nowhere else, they must be equal up to a scalar in the coordinate ring of the plane, e.g. they are equal up to a scalar modulo $h$.

Let $h(\mathbf{P})=\alpha \times X+\beta \times Y+\gamma \times Z+\delta \times T$, where $\mathbf{P}=$ $[X, Y, Y, T]^{T}$. The theorem 8 provides $s=\left(\begin{array}{c}3+d \\ d\end{array}\right)$ equations on $k, \alpha, \beta, \gamma, \delta,\left(r_{i}\right)_{1<i<d-1}$, where $\left(r_{i}\right)_{i}$ are the coefficients of $r$. Now we proceed to show an explicit way to perform the elimination of the auxiliary unknowns: $k,\left(r_{i}\right)$. Let $S$ be the surface, whose equation is $\Sigma=\Delta_{1}+k \Delta_{2}=0$. The points $\mathbf{P}$ that lie on the plane $\mathbf{H}$ are characterized by the fact that when regarded as points of $S$, their tangent planes are exactly $\mathbf{H}$. This is expressed by the following system of equations: 


$$
\left\{\begin{array}{c}
h(\mathbf{P})=0 \\
\left(\beta \frac{\partial \Sigma}{\partial X}-\alpha \frac{\partial \Sigma}{\partial Y}\right)(\mathbf{P})=0 \\
\left(\gamma \frac{\partial \Sigma}{\partial X}-\alpha \frac{\partial \Sigma}{\partial Z}\right)(\mathbf{P})=0 \\
\left(\delta \frac{\partial \Sigma}{\partial X}-\alpha \frac{\partial \Sigma}{\partial T}\right)(\mathbf{P})=
\end{array}\right.
$$

On the other hand, on the plane $\Sigma(\mathbf{P})=\Delta_{1}(\mathbf{P})+$ $k \Delta_{2}(\mathbf{P})=0$. Therefore $k=-\frac{\Delta_{1}(\mathbf{P})}{\Delta_{2}(\mathbf{P})}$ for any $\mathbf{P}$ on the plane that is not located on the curve itself. Therefore we get the following system:

$\left\{\begin{array}{c}h(\mathbf{P})=0 \\ \left(\beta\left(\Delta_{2} \frac{\partial \Delta_{1}}{\partial X}-\Delta_{1} \frac{\partial \Delta_{2}}{\partial X}\right)-\alpha\left(\left(\Delta_{2} \frac{\partial \Delta_{1}}{\partial Y}-\Delta_{1} \frac{\partial \Delta_{2}}{\partial Y}\right)\right)(\mathbf{P})=\right. \\ \left(\gamma\left(\Delta_{2} \frac{\partial \Delta_{1}}{\partial X}-\Delta_{1} \frac{\partial \Delta_{2}}{\partial X}\right)-\alpha\left(\left(\Delta_{2} \frac{\partial \Delta_{1}}{\partial Z_{Z}}-\Delta_{1} \frac{\partial \Delta_{2}}{\partial Z}\right)\right)(\mathbf{P})=\right. \\ \left(\delta\left(\Delta_{2} \frac{\partial \Delta_{1}}{\partial X}-\Delta_{1} \frac{\partial \Delta_{2}}{\partial X}\right)-\alpha\left(\left(\Delta_{2} \frac{\partial \Delta_{1}}{\partial T}-\Delta_{1} \frac{\partial \Delta_{2}}{\partial T}\right)\right)(\mathbf{P})=\right.\end{array}\right.$

Since the plane we are looking for does not pass through the point $[0,0,0,1]^{T}$ which is the first camera center, $\delta$ can be normalized to 1 . Thus for a point $\mathbf{P}$ on the plane, we have: $T=-(\alpha X+\beta Y+\gamma Z)$. By substituting this expression of $T$ into the previous system, we get a new system that vanishes over all values of $(X, Y, Z)$. Therefore its coefficients must be zero. This provides us with a large set of equations on $(\alpha, \beta, \gamma)$, that can be used to refine the solution obtained by the homography based approach. Moreover once the plane is computed, its intersection with one of the image cones can be computed, the outcome being the equation of the curve on the plane. For that purpose, one has to eliminate $T$ between the two equations: $\alpha X+\beta Y+\gamma Z+T=0$ and $f_{1}\left(\mathbf{M}_{1} \mathbf{P}\right)=0$. When a projective calibration only is known, then the first camera matrix is $[\mathbf{I} ; \mathbf{0}]$. Thus this yields immediately the equation of the curve on $\mathbf{H}$.

\subsection{Reconstruction in the Dual Space}

As above, let $X$ be the curve in space, that we want to reconstruct. Let $X^{\star}$ be the dual variety of $X$, that is, the set of planes tangent to $X$. Since $X$ is supposed not to be a line, the dual variety $X^{\star}$ must be a hypersurface of the dual space [18]. Hence let $\Upsilon$ be a minimal degree polynomial that represents $X^{\star}$. Our first concern is to determine the degree of $\Upsilon$.

Proposition 11 The degree of $\Upsilon$ is $m$, that is, the common degree of the dual image curves.

Proof: Since $X^{\star}$ is a hypersurface of $\mathbb{P}^{3 \star}$, its degree is the number of points where a generic line in $\mathbb{P}^{3 \star}$ meets $X^{\star}$. By duality it is the number of planes in a generic pencil that are tangent to $X$. Hence it is the degree of the dual image curve. Another way to express the same fact is the observation that the dual image curve is the intersection of $X^{\star}$ with a generic plane in $\mathbb{P}^{3 \star}$. Note that this provides a new proof that the degree of the dual image curve is constant for a generic position of the camera center.

The reconstruction problem can be regarded in two different ways leading to two different applications. In the first approach, we get an algorithm to reconstruct a curve from a set of views, while in the second approach we show how to recover an arbitrary trajectory of a moving point from a moving camera.

For the reconstruction of $X^{*}$ from multiple view, we will need to consider the mapping from a line 1 of the image plane to the plane that it defines with the camera center. Let $\mu: \mathbf{l} \mapsto \mathbf{M}^{T} \mathbf{l}$ denote this mapping [11]. There exists a Olink involving $\Upsilon, \mu$ and $\phi$, the polynomial of the dual image ocurve: $\Upsilon(\mu(\mathbf{l}))=0$ whenever $\phi(\mathbf{l})=0$. Since these two opolynomials have the same degree (because $\mu$ is linear) and $\phi$ is irreducible, there exist a scalar $\lambda$ such that

$$
\Upsilon(\mu(\mathbf{l}))=\lambda \phi(\mathbf{l})
$$

for all lines $\mathbf{l} \in \mathbb{P}^{2 \star}$. Eliminating $\lambda$, we get $\frac{(m+2)(m+1)}{2}-1$ linear equations on $\Upsilon$. Since the number of coefficients in $\Upsilon$ is $\frac{(m+3)(m+2)(m+1)}{6}$, we can state the following result:

Proposition 12 The reconstruction in the dual space can be done linearly using at least $k \geq \frac{m^{2}+6 m+11}{3(m+3)}$ views.

Proof: The least number of views must satisfy $k\left(\frac{(m+2)(m+1)}{2}-1\right) \geq \frac{(m+3)(m+2)(m+1)}{6}-1$.

The lower bounds on the number of views $k$ for few examples are given below:

1. for a conic locus, $k \geq 2$,

2. for a rational cubic, $k \geq 3$,

3. for an elliptic cubic, $k \geq 4$,

4. for a rational quartic, $k \geq 4$,

5. for a elliptic quartic, $k \geq 4$.

Moreover it is worth noting that the fitting of the dual image curve is not necessary. It is sufficient to extract tangents to the image curves at distinct points. Each tangent 1 contributes to one linear equation on $\Upsilon$ : $\Upsilon(\mu(\mathbf{l}))=0$. However one cannot obtain more than $\frac{(m+2)(m+1)}{2}-1$ linearly independent equations per view.

Consider next a single moving camera viewing a moving point. Assume that at a time $t$, only the tangent of the trajectory is extracted from the image. As mentioned previously, each such tangent 1 yields a linear constraint on $\Upsilon$.

Proposition 13 The reconstruction of the trajectory of a moving point can be done by tangential measurements from a moving camera using at least $k \geq \frac{(m+3)(m+2)(m+1)}{6}-1$ images. 
Note that in the case of conics were presented in [38]. Here we summarize the minimal value of $k$ in few cases:

1. for a moving point on a conic locus, $k \geq 9$,

2. for a moving point on a rational cubic, $k \geq 34$,

3. for a moving point on an elliptic cubic, $k \geq 83$,

4. for a moving point on a rational quartic, $k \geq 83$.

\subsection{Reconstruction in $\mathbb{G}(1,3)$}

The spatial curve $X$ admits $\Gamma$ as a Chow polynomial. Let $d$ be the common degree of $X$ and $\Gamma$. Let $f$ be the polynomial defining the image curve, $Y$. Consider the mapping that associates to an image point its optical ray: $\nu: \mathbf{p} \mapsto \widehat{\mathrm{M}} \mathbf{p}$, where $\widehat{\mathbf{M}}$ is a $3 \times 6$ matrix, which entries are polynomials functions of $\mathbf{M}$ [11]. Hence the polynomial $\Gamma(\nu(\mathbf{p}))$ vanishes whenever $f(\mathbf{p})$ does. Since they have same degree and $f$ is irreducible, there exists a scalar $\lambda$ such as for every point $\mathbf{p} \in \mathbb{P}^{2}$, we have:

$$
\Gamma(\nu(\mathbf{p}))=\lambda f(\mathbf{p}) .
$$

This yields $\left(\begin{array}{c}d+2 \\ d\end{array}\right)-1$ linear equations on $\Gamma$.

Hence a similar statement to that in Proposition 12 can be made:

Proposition 14 The reconstruction in $\mathbb{G}(1,3)$ can be done linearly using at least $k \geq \frac{1}{6} \frac{d^{3}+5 d^{2}+8 d+4}{d}$ views.

For some examples, below are the minimal number of views for a linear reconstruction of the curve in $\mathbb{G}(1,3)$ :

1. for a conic locus, $k \geq 4$,

2. for a cubic, $k \geq 6$,

3. for a quartic, $k \geq 8$.

As in the case of reconstruction in the dual space, it is not necessary to explicitly compute $f$. It is enough to pick points on the image curve. Each point yields a linear equation on $\Gamma: \Gamma(\nu(\mathbf{p}))=0$. However for each view, one cannot extract more than $\frac{1}{2} d^{2}+\frac{3}{2} d$ independent linear equations.

Consider next a single moving camera viewing a moving point. As mentioned previously, each point contributes one linear equations on $\Gamma$. Hence the following result follows.

Proposition 15 The recovery of the trajectory of a moving point by point based measurements from a single moving camera can be done linearly using at least $k \geq \frac{1}{12} d^{4}+$ $\frac{2}{3} d^{3}+\frac{23}{12} d^{2}+\frac{7}{3} d+1$ such measurements.

The lower bounds on $k$ for few examples:
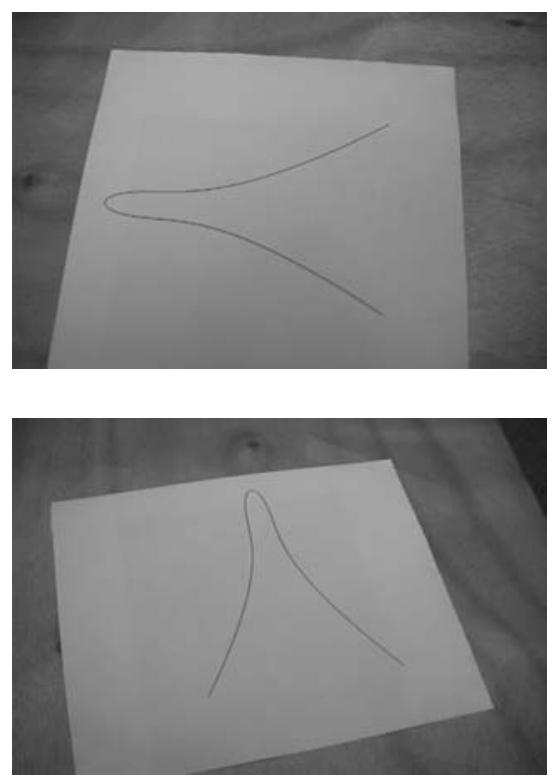

Figure 5: The two images of a cubic curve.

1. for a moving point on a conic locus, $k \geq 20$,

2. for a moving point on a cubic, $k \geq 50$,

3. for a moving point on a quartic, $k \geq 105$.

\section{Experiments}

\subsection{Experiments on real images}

\subsubsection{Homography recovery from a single planar curve by point extraction}

In the first experiment, we consider the problem of recovering the homography matrix induced by a planar cubic across two images (see Figure 5) using the method described in 3.1.4 (i.e. without prior knowledge of the epipolar geometry). The cubic equations of the image curves were recovered by least-squares fitting. The recovered homography was then used to re-project the curve from one image onto the other. The reprojection error was at subpixel values (see Figure 6).

\subsubsection{Recovering homography from planar curves cor- respondences}

Given two images of the same curve of order 4 (see Figure 7) and the epipolar geometry, we start by computing the plane and the homography matrix, using the algebraic approach described in 4.1. In order to refine the solution we place it into the final system, obtained at the end of 4.2.2 and 

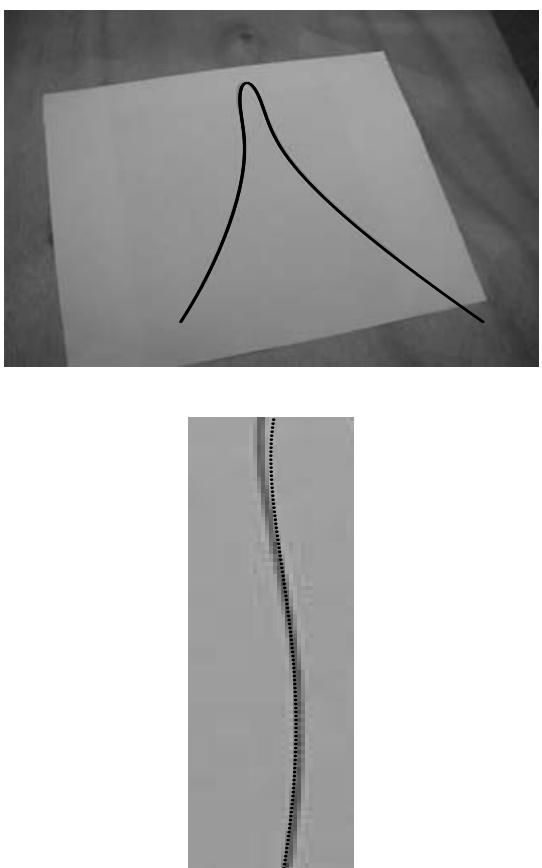

Figure 6: The reprojected curve is overlaid on the second image cubic. The bottom display shows an enlarged section of the curve and the overlaid reprojected curve - the error is at subpixel values.
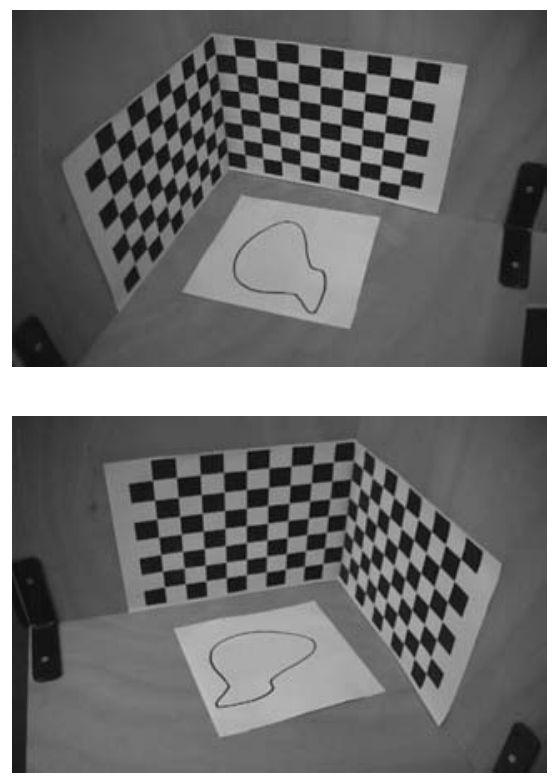

Figure 7: The curves of order 4 as an input of the reconstruction algorithm.

then use a local optimization algorithm. To demonstrate the accuracy of the algorithm, the reprojection of the curve in the second image is shown in the figure 8 . The $3 \mathrm{D}$ rendering of the correct solution is shown 9 .

Finally, the equation of the correct solution on its plane is given by:

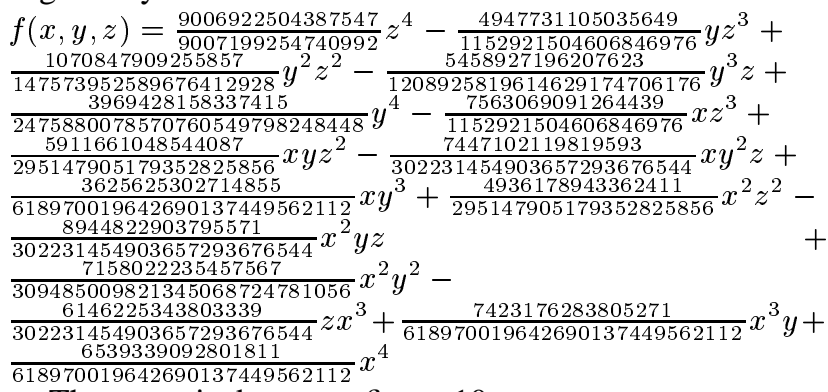

The curve is drawn on figure 10 .

\subsubsection{Epipolar geometry from points and conic corre- spondences}

We proceeded to the recovery of the epipolar geometry from conics and points correspondences extracted from real images. The extraction has been done manually and the conics were fitted by classical least square optimization.

The recovery of the epipolar geometry has been done using four conics and 1 point. First the fundamental matrix is computed using three conics and 1 point, which leads to a finite number of solutions (see theorem 3 ). The computation is too intense for the standard computer algebra packages. 

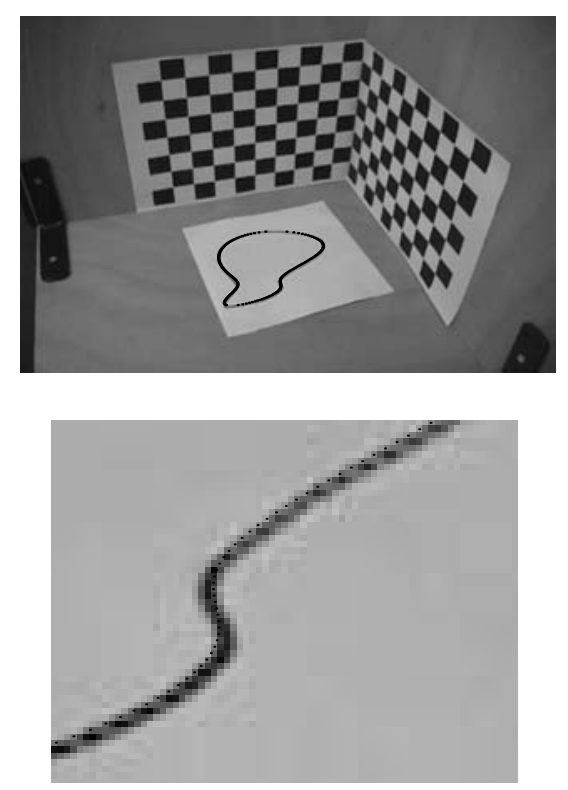

Figure 8: Reprojection of the curve onto the second image.

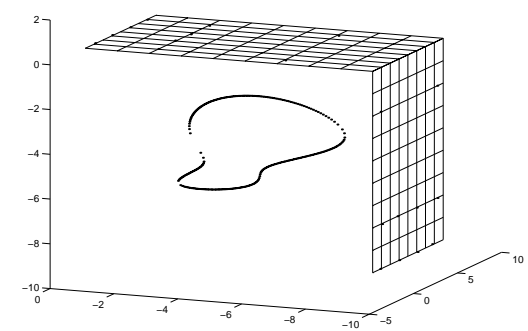

Figure 9: The curve of order 4 as an output of the reconstruction algorithm.

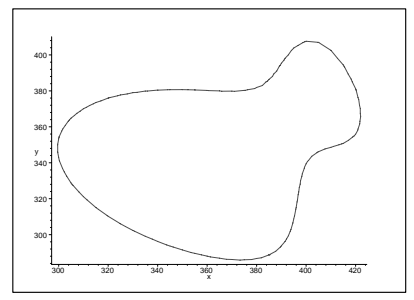

Figure 10: The original curve.
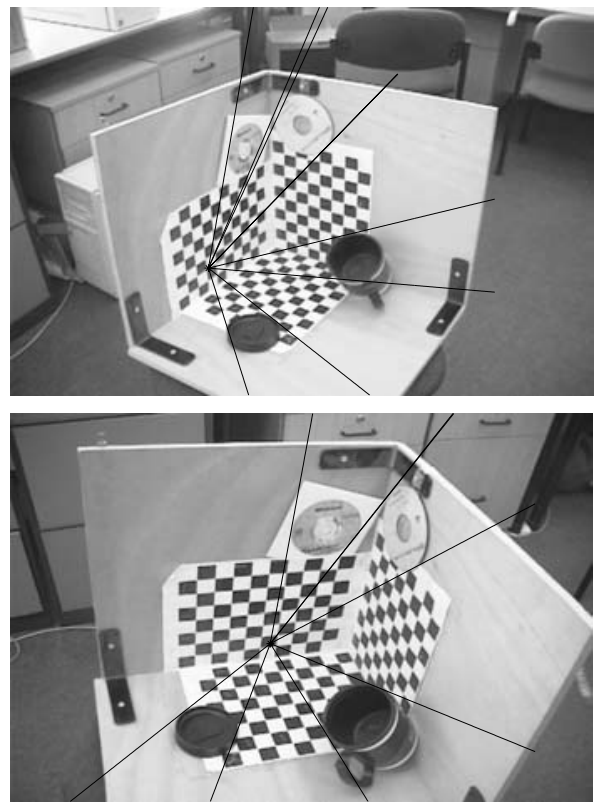

Figure 11: The two images that were used. The epipoles and the corresponding epipolar lines tangents to the conics are drawn on the images.

We have found that Fast Gb ${ }^{6}$, a powerful software tool for Gröbner basis computation, introduced by J.C. Faugere $[12,13]$ is one of the few packages that can handle this kind of computation. Then the additional conic is used to select the right solution.

The images used for the experiments together with results and comments are presented in figure 11

\subsubsection{D reconstruction using the Grassmannian $\mathbb{G}(1,3)$}

For the next experiment, we consider seven images of an electric wire - one of the views is shown in figure 12 and the image curve after segmentation and thinning is shown in figure 13. Hence for each of the images, we extracted a set of points lying on the thread. No fitting is performed in the image space. For each image, the camera matrix is calculated using the calibration pattern. Then we proceeded to compute the Chow polynomial $\Gamma$ of the curve in space. The curve $X$ has degree 3 . Once $\Gamma$ is computed, a reprojection is easily performed, as shown in figure 14 .

\footnotetext{
${ }^{6}$ Logiciel conçu et réalisé au laboratoire LIP6 de l'université Pierre et Marie CURIE.
} 


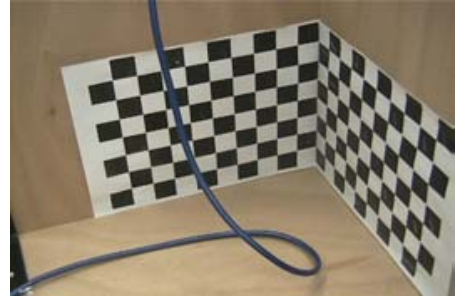

Figure 12: An electric thread.

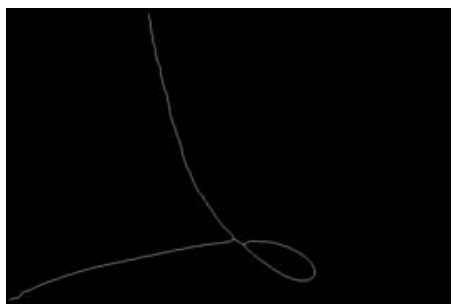

Figure 13: An electric thread after segmentation and thinning.

\subsection{Experiments on synthetic data}

\subsubsection{Recovering epipolar geometry from conics corre- spondences}

We compute the fundamental matrix from 3 conics and 2 points in a synthetic experiment. Once again the computation were made by FGb. The conics in the first image are:

$$
\begin{aligned}
& f 1(x, y, z)=x^{2}+y^{2}+9 z^{2} \\
& g 1(x, y, z)=4 x^{2}+y^{2}+81 z^{2} \\
& h 1(x, y, z)=(4 x+y) x+(x-1 / 2 z) y+(-1 / 2 y+z) z
\end{aligned}
$$

The conics in the second image are:

$f 2(x, y, z)=-\frac{1}{100(-4+\sqrt{3})^{2}}\left(-1900 x^{2}+\right.$ $800 x^{2} \sqrt{3}-1309 y^{2}+400 y^{2} \sqrt{3}+9820 y z \sqrt{3}-$ $\left.16000 y z-72700 z^{2}+40000 z^{2} \sqrt{3}\right)$ $g 2(x, y, z)=\frac{1}{400(4489+400 \sqrt{3})^{2}}\left(33036473600 x^{2}+\right.$ $5732960000 x^{2} \sqrt{3}+332999600 x y \sqrt{3}-$ $214463200 x y-73852000 x z-1384952000 x z \sqrt{3}+$ $9091399981 y^{2}+1771266080 y^{2} \sqrt{3}-$

$16090386780 y z \sqrt{3}+10160177600 y z+556496242300 z^{2}+$ $\left.141582592000 z^{2} \sqrt{3}\right)$

$h 2(x, y, z)=-\frac{1}{400(-561+38 \sqrt{3})^{2}}\left(-519504000 x^{2}+\right.$ $48311700 z^{2}-125749120 x y \sqrt{3}+43249920 x y-$ $254646400 x z \sqrt{3}-6553140 y z \sqrt{3}+$ $56456040 y z+68848000 x^{2} \sqrt{3}+1279651200 x z-$ $\left.272267400 z^{2} \sqrt{3}+2522418 y^{2} \sqrt{3}-298209 y^{2}\right)$

Given just the constraints deduced from the conics, the system defines, as expected, a one-dimensional variety in $\mathbb{P}^{2} \times \mathbb{P}^{8}$. When just one point is introduced, we get a zerodimensional variety, whose degree is 516 . When two points

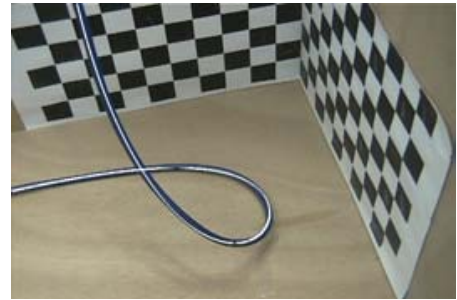

Figure 14: Reprojection on a new image.

are introduced, the system reduces to the following:

$$
\left\{\begin{array}{c}
F[1,1]=F[2,2]=F[2,3]=F[3,2]=F[3,3]=0 \\
F[3,1]+(\sqrt{3}-1) F[1,3]=0 \\
10 F[2,1]+(\sqrt{3}-1) F[1,3]=0 \\
10 F[1,2]+(\sqrt{3}-2) F[1,3]=0 \\
133813 F[1,3]^{2}-20600 \sqrt{3}-51100=0
\end{array}\right.
$$

Then it is easy to get the right answer for the fundamental matrix:

$$
\left[\begin{array}{ccc}
0 & -\frac{-2+\sqrt{3}}{\sqrt{511-206 \sqrt{3}}} & 10 \frac{1}{\sqrt{511-206 \sqrt{3}}} \\
-\frac{-1+\sqrt{3}}{\sqrt{511-206 \sqrt{3}}} & 0 & 0 \\
-10 \frac{-1+\sqrt{3}}{\sqrt{511-206 \sqrt{3}}} & 0 & 0
\end{array}\right]
$$

\subsubsection{Recovering epipolar geometry from spatial curves correspondences}

We proceed to the computation of the epipolar geometry from a rational cubic and two conics. The curves are randomly chosen, as well as the camera.

Hence the cubic is defined by the following system:

$226566665956452626 Z X-1914854993236086169 Z T-$ $791130248041963297 Y Z-1198609868087508022 Z^{2}+$ $893468169675527814 X T+285940501848919422 T^{2}-$ $179632615056970090 Y T+277960038226472656 Y^{2}=0$

$555920076452945312 X Y+656494420457765614 Z X-$ $1755155973545148735 Y Z-1749154450800074954 Z^{2}+$ $984240461094724954 X T-61309565864179510 Y T-$ $1802588912007356295 Z T+291319745776795474 T^{2}=0$

$1111840152905890624 X^{2}-2905335341664005486 Z X-$ $793850352563738017 Y Z+1286890161434843658 Z^{2}+$ $1713207647519936006 X T-248798847306328202 Y T-$ $2942349361064284313 Z T+398814386951585134 T^{2}=0$

The first and the second conic are respectively defined by:

$$
\begin{aligned}
& 25 X+9 Y+40 Z+61 T=0 \\
& 40 X^{2}-78 X Y+62 Z X+11 X T+88 Y^{2}+ \\
& Y Z+30 Y T+81 Z^{2}-5 Z T-28 T^{2}=0
\end{aligned}
$$




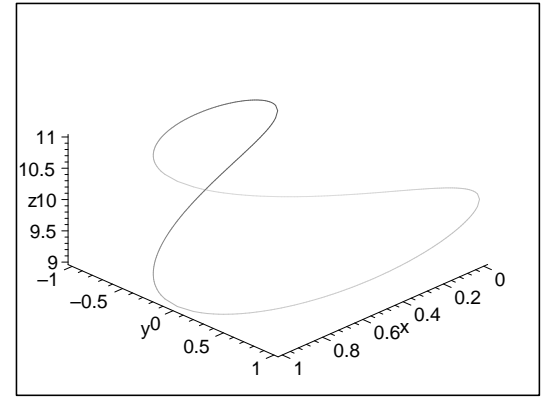

Figure 15: A spatial quartic

and

$$
\begin{aligned}
& 4 X-11 Y+10 Z+57 T=0 \\
& -82 X^{2}-48 X Y-11 Z X+38 X T-7 Y^{2}+ \\
& 58 Y Z-94 Y T-68 Z^{2}+14 Z T-35 T^{2}=0
\end{aligned}
$$

The camera matrices are given by:

$$
\begin{aligned}
\mathbf{M}_{1} & =\left[\begin{array}{cccc}
-87 & 79 & 43 & -66 \\
-53 & -61 & -23 & -37 \\
31 & -34 & -42 & 88
\end{array}\right] \\
\mathbf{M}_{2} & =\left[\begin{array}{cccc}
-76 & -65 & 25 & 28 \\
-61 & -60 & 9 & 29 \\
-66 & -32 & 78 & 39
\end{array}\right]
\end{aligned}
$$

Then we form the Extended Kruppa's Equations for each curve. From a computational point of view, it is crucial to enforce the constraint that each $\lambda$ is different from zero. Mathematically this means that the computation is done in the localization with respect to each $\lambda$.

As expected, we get a zero-dimension variety which degree is one. Thus there is a single solution to the epipolar geometry given by the following fundamental matrix:

$$
\mathbf{F}=\left[\begin{array}{ccc}
-\frac{511443}{13426} & -\frac{2669337}{13426} & -\frac{998290}{6713} \\
\frac{84845}{2329} & \frac{23737631}{114121} & \frac{14061396}{114121} \\
\frac{1691905}{228242} & \frac{3426650}{114121} & \frac{8707255}{228242}
\end{array}\right]
$$

\subsubsection{Reconstruction of spatial curves}

We start with a synthetic experiment followed later by a real image one. Consider the curve $X$, drawn in figure 15, defined by the following equations:

$$
\begin{gathered}
F_{1}(x, y, z, t)=x^{2}+y^{2}-t^{2} \\
F_{2}(x, y, z, t)=x t-(z-10 t)^{2}
\end{gathered}
$$

The curve $X$ is smooth and irreducible, and has degree 4 and genus 1 . We define two camera matrices:

$$
\begin{aligned}
& \mathbf{M}_{1}=\left[\begin{array}{cccc}
1 & 0 & 0 & 5 \\
0 & 0 & 1 & -2 \\
0 & -1 & 0 & -10
\end{array}\right] \\
& \mathbf{M}_{2}=\left[\begin{array}{cccc}
1 & 0 & 0 & -10 \\
0 & 0 & -1 & 0 \\
0 & 1 & 0 & -10
\end{array}\right]
\end{aligned}
$$

The reconstruction of the curve from the two projections has been made in the point space, using $\mathbf{F G b}{ }^{7}$, a powerful software tool for Gröbner basis computation [12, 13]. As expected there are two irreducible components. One has degree 4 and is the original curve, while the second has degree 12 .

\section{Summary and Discussion}

In this paper we have focused on general algebraic curves as the building blocks from which the camera geometries are to be recovered and as the scene building blocks for the purpose of reconstruction from multiple views. The new results derived in this paper include:

1. Extended Kruppa's equations for the recovery of epipolar geometry from two projections of algebraic curves.

2. Dimension analysis for the minimal number of algebraic curves required for a solution of the epipolar geometry.

3. Homography recovery from two views of a general planar curve, when the epipolar geometry is either known or not.

4. The reconstruction from two views of an irreducible curve of degree $d$ is a curve which contains two irreducible components one of degree $d$ and the other of degree $d(d-1)$ - a result that leads to a unique reconstruction of the original curve, for $d>2$.

5. Formula for the minimal number of views required for the reconstruction of the dual curve.

6. Formula for the minimal number of views required for the reconstruction of the curve representation in $\mathbb{G}(1,3)$.

Most of the algorithms presented in this work lead to solving a system of polynomial equations. As previously mentioned, there exist two main approaches to handle this problem: (i) computing a Gröbner basis of the ideal defined by the equations, (ii) processing in the dual space via the

\footnotetext{
${ }^{7}$ Logiciel conçu et réalisé au laboratoire LIP6 de l'université Pierre et Marie CURIE.
} 
computation of resultants (see [5, 6] for a detailed presentation). There exists a third method, known as the homotopy method, whose field of applications is broader than the resolution of polynomial systems [1]. However it is generally admitted that the symbolic methods, namely those based on Gröbner basis or resultants, provide better results.

Note that numerical optimization tools like NewtonRaphson or Levenberg-Marquet optimization are not considered here because (i) zero-dimensional polynomial systems which are not overdetermined have more than one root and these optimization methods are designed to extract a single solutions, (ii) the convergence to a solution with these tools is well behaved only when one starts in a small enough neighborhood of the solution.

The use of symbolic tools (either Gröbner basis or resultant) for computer vision applications is not without challenges. First, symbolic computations require large amounts of available computational and memory resources. There is the issue of computational efficiency, scalability to large problems and the questions of effectiveness in the presence of measurement errors. The full answer to these questions is far beyond the scope of this work. The field of symbolic computations for solving polynomial systems is a very active field of research where major progress has been made in the past decade $[6,17,42]$. For example, throughout this paper, the experiments were performed with one of the latest symbolic tools "FastGB" developed by Jean-Charles Faugere for efficient and robust Gröbner basis computation. With those latest tools, such as FastGB, one can achieve a high degree of scalability and efficiency in the computations.

Finally the problem of the sensitivity to noise is related to perturbation theory. It is necessary to note that since the computations are symbolic, they do not add any perturbation to the solution. Therefore, as opposed to numerical methods, there is no additional error due to possible truncation during the computations. However, there is very little research on measurement error sensitivity and their propagation throughout the symbolic computations. Such research would be of great interest to the computer vision community, however, this topic is largely open. Nevertheless, a first step in this direction has been done by the introduction of a hybrid of symbolic and numeric computations, especially for the case of zero-dimensional system (which is the case of interest in vision) solved by resultant based methods $[42,32]$.

\section{Acknowledgment}

We thank Michael Fryers and Mina Teicher for their fruitful help. As well, we express our gratitude to Jean-Charles Faugere for giving us access to his powerful system FGb.

\section{References}

[1] E. Allgower and K.Georg. Numerical continuation method, An introduction. Number 13 in Computational Mathematics, Springer-Verlag, 1990.

[2] K. Astrom and F. Kahl. Motion Estimation in Image Sequences Using the Deformation of Apparent Contours. In IEEE Transactions on Pattern Analysis and Machine Intelligence, 21(2), February 1999.

[3] M. Barnabei, A. Brini and G.C. Rota On the exterior calculus of invariant theory. Journal of Algebra, 96, 120-160(1985)

[4] R. Berthilson, K. Astrom and A. Heyden. Reconstruction of curves in $\mathcal{R}^{3}$, using Factorization and Bundle Adjustment. In IEEE Transactions on Pattern Analysis and Machine Intelligence, 21(2), February 1999.

[5] D. Cox, J. Little and D. O'shea. Ideals, Varieties and Algorithms, Second Edition, Springer-Verlag, 1997.

[6] D. Cox, J. Little and D. O'shea. Using Algebraic Geometry, Springer-Verlag, 1998.

[7] D. Eisenbud. Commutative Algebra with a view toward algebraic geometry. Springer-Verlag, 1995.

[8] D. Eisenbud and J. Harris. The Geometry of Schemes. Springer-Verlag, 2000

[9] O.D. Faugeras Three-Dimensional Computer Vision, A geometric approach. MIT Press, 1993.

[10] O.D. Faugeras and Q.T. Luong The geometry of multiple images. MIT Press, 2001.

[11] 0.D. Faugeras and T. Papadopoulo. Grassman-Cayley algebra for modeling systems of cameras and the algebraic equations of the manifold of trifocal tensors. Technical Report INRIA 3225, July 1997.

[12] J.C. Faugere. Computing Grobner basis without reduction to zero $\left(F_{5}\right)$. Technical report, LIP6, 1998.

[13] J.C. Faugere. A new efficient algorithm for computing Grobner basis $\left(F_{4}\right)$.

[14] D. Forsyth, Recognizing algebraic surfaces from their outlines.

[15] W. Fulton Algebraic Curves.

[16] Cross and A. Zisserman, Quadric Reconstruction from DualSpace Geometry, 1998.

[17] G.M. Greuel and G. Pfister, A Singular Introduction to Commutative Algebra. Springer-Verlag, 2002.

[18] J. Harris Algebraic Geometry, a first course. SpringerVerlag, 1992.

[19] J. Harris and Griffith Principle of algberaic geometry. 
[20] R. Hartley and A. Zisserman Multiple View Geometry in computer vision. Cambridge Univeristy Press, 2000.

[21] R. Hartshorne. Algebraic Geometry. Springer-Verlag, 1977.

[22] D.P. Huttenlocher, G.A. Klanderman and W.J. Rucklidge Comparing images using the Hausdorff distance. In IEEE Transactions on Pattern Analysis and Machine Intelligence, 15(9), September 1993.

[23] F. Kahl and A. Heyden. Using Conic Correspondence in Two Images to Estimate the Epipolar Geometry. In Proceedings of the International Conference on Computer Vision, 1998.

[24] J.Y. Kaminski Multiple-view Geometry of Algebraic Curves. Phd dissertation, The Hebrew University Of Jerusalem, June 2001.

[25] J.Y. Kaminski and A. Shashua. On Calibration and Reconstruction from Planar Curves. In Proceedings European Conference on Computer Vision, 2000.

[26] J.Y. Kaminski, M.Fryers, A.Shashua and M.Teicher. Multiple View Geometry Of (Non-Planar) Algebraic Curves. In Proceedings of the International Conference on Computer Vision, 2001.

[27] S. Lang Algebra Addison-Wesley Publishing Company, Inc.

[28] Q.T Luong and T. Vieville. Canonic Representations for the Geometries of Multiple Projective Views. In Proceedings European Conference on Computer Vision, 1994.

[29] S.D. Ma and X. Chen. Quadric Reconstruction from its Occluding Contours. In Proceedings International Conference of Pattern Recognition, 1994.

[30] S.D. Ma and L. Li. Ellipsoid Reconstruction from Three Perspective Views. In Proceedings International Conference of Pattern Recognition, 1996.

[31] S.J. Maybank and O.D. Faugeras A theory of self-calibration of a moving camera. International Journal of Computer Vision, 8(2):123-151, 1992.

[32] B. Mourrain and Ph. Trébuchet Algebraic methods for numerical solving. In Proceedings of the 3rd International Workshop on Symbolic and Numeric Algorithms for Scientific Computing'01, pp. 42-57, 2002.

[33] T. Papadopoulo and O. Faugeras Computing structure and motion of general $3 \mathrm{~d}$ curves from monocular sequences of perspective images. In Proceedings European Conference on Computer Vision, 1996.

[34] T. Papadopoulo and O. Faugeras Computing structure and motion of general $3 \mathrm{~d}$ curves from monocular sequences of perspective images. Technical Report 2765, INRIA, 1995.

[35] L. Quan. Conic Reconstruction and Correspondence from Two Views. In IEEE Transactions on Pattern Analysis and Machine Intelligence, 18(2), February 1996.
[36] E. Reyssat Quelques Aspecets des Surfaces de Riemann. Bikhauser, 1989.

[37] C. Schmid and A. Zisserman. The Geometry and Matching of Curves in Multiple Views. In Proceedings European Conference on Computer Vision, 1998.

[38] D. Segal and A. Shashua 3D Reconstruction from Tangentof-Sight Measurements of a Moving Object Seen from a Moving Camera. In Proceedings European Conference on Computer Vision, 2000

[39] J.G. Semple and G.T. Kneebone. Algebraic Curves. Oxford University Press, 1959.

[40] A. Shashua and N. Navab. Relative Affine Structure: Canonical Model for 3D from 2D Geometry and Applications. IEEE Transactions on Pattern Analysis and Machine Intelligence, 18(9):873-883, 1996.

[41] A. Shashua and S. Toelg The Quadric Reference Surface: Theory and Applications. International Journal of Computer Vision, 23(2):185-198, 1997.

[42] B. Sturmfels, Solving Systems of Polynomials Equations, American Mathematical Society, 2002.

[43] R.J. Walker Algebraic Curves Princeton University Press, 1950.

\section{A. Technical background}

This section provides a brief introduction to algebraic geometry, and algebraic curves in particular, necessary for the overall understanding of our work.

The ground field is assumed to be the field of complex number. This is important from a theoretical point of view, since the field of complex number is algebraically closed. However from a practical point of view, we are interested in the real locus of all varieties. Therefore all the algorithms must eventually extract only the real points.

\section{A.1. Algebraic variety}

\section{A.1.1 Affine variety}

Definition 1 Given a polynomial $F$ in the polynomial ring $\mathbb{C}\left[X_{1}, \ldots ., X_{n}\right]$, we say that a point $P$ in the $n-$ dimensional affine space $\mathbb{A}^{n}$, regarded as a $m$-tuple $\left(P_{1}, \ldots ., P_{n}\right)$, is a zero of $F$ if $F(P)=0$.

Definition 2 An algebraic variety in the affine space $\mathbb{A}^{n}$ is a subset of $\mathbb{A}^{n}$ being the common zeros of a family of polynomial $\left\{F_{i}\right\}$ :

$$
Z\left(\left\{F_{i}\right\}\right)=\left\{P \in \mathbb{A}^{n} \mid F_{i}(P)=0 \text { for all } i\right\}
$$


It is clear that the variety will not be affected if we add to the family all the combinations: $\sum_{i} G_{i} F_{i}$, where all but a finite number of $G_{i}$ are zeros. Hence a variety of $\mathbb{A}^{n}$ is always defined by the ideal ([27]) generated by its defining family. We immediately deduce that:

Proposition 16 The union of two varieties is a variety. The intersection of any family of varieties is a variety. The empty set and the whole space are varieties.

Then the varieties are the closed sets of a topology called the Zariski topology.

Given an ideal $I$ in $\mathbb{C}\left[X_{1}, \ldots, X_{n}\right]$, we shall denote by $Z(I)$ the variety it defines. Since the polynomial ring is noetherian, we get:

Proposition 17 A variety is always defined by a finite set of equations, namely the generators of the ideal, which defines the variety.

As each ideal defines a variety, any subset $S$ of $\mathbb{A}^{n}$ defines an ideal as follows:

$$
I(S)=\left\{F \in \mathbb{C}\left[X_{1}, \ldots, X_{n}\right] \mid \forall P \in S, F(P)=0\right\}
$$

There is a relationship between ideals of $\mathbb{C}\left[X_{1}, \ldots, X_{n}\right]$ and varieties of $\mathbb{A}^{n}$. The following properties can be proven:

Proposition 18 1. If $S_{1} \subset S_{2}$ are two subsets of $\mathbb{A}^{n}$, then $I\left(S_{2}\right) \subset I\left(S_{1}\right)$.

2. If $I_{1} \subset I_{2}$ are two ideals of $\mathbb{C}\left[X_{1}, \ldots, X_{n}\right]$, then $Z\left(I_{2}\right) \subset Z\left(I_{1}\right)$.

3. For any two subsets $S_{1}$ and $S_{2}$ of $\mathbb{A}^{n}$, we have: $I\left(S_{1} \cup\right.$ $\left.S_{2}\right)=I\left(S_{1}\right) \cap I\left(S_{2}\right)$

4. For any ideal $I \subset \mathbb{C}\left[X_{1}, \ldots, X_{n}\right], I(Z(I))=$ $\sqrt{I}$, the radical of $I$, defined by $\sqrt{I}=\{F \in$ $\left.\mathbb{C}\left[x_{1}, \ldots, x_{n}\right] \mid \exists r, F^{r} \in I\right\}$.

5. For any subset $S \in \mathbb{A}^{n}, Z(I(S))=\bar{S}$, the closure of $S$.

Note that the fourth point is a direct consequence of the Hilbert's Nullstellensatz:

Theorem 9 When the ground field is algebraically closed (which is the case for complex varieties), the following holds. Let $I$ be an ideal of $\mathbb{C}\left[X_{1}, \ldots, X_{n}\right]$, and let $F \in$ $\mathbb{C}\left[X_{1}, \ldots, X_{n}\right]$ which vanishes at all points of $Z(I)$. Then $F^{r} \in I$ for some integer $r>0$.

We end this very short introduction by defining irreducibity and dimension.
Definition 3 A variety is said to be irreducible, if it cannot be expressed as the union of two non-empty proper subvarieties.

Definition 4 The dimension of a variety $X$ if the supremum of all integers $k$ such that there exists a chain $Z_{0} \subset Z_{1} \subset$ $\ldots . \subset Z_{k}$ of distinct irreducible sub-varieties of $X$.

\section{A.1.2 Projective variety}

As we defined an affine variety to be a subset of an affine space $\mathbb{A}^{n}$, defined by polynomials equations, we shall define a projective variety to be a subset of a projective space $\mathbb{P}^{n}$ defined by homogeneous polynomials.

The properties of projective varieties are very similar to those of affine varieties.

\section{A.2. Algebraic planar curves}

\section{A.2.1 Definitions}

Definition 5 A polynomial $f$ is said to be square-free if it cannot be written as a product like: $f=g^{2} h$, where $g$ and $h$ are non constant polynomials.

Definition 6 A planar algebraic curve $\mathcal{C}$ is a subset of points, whose projective coordinates satisfy an homogeneous square-free polynomial equation: $f(x, y, z)=0$. The degree of $f$ is called the order or degree of $\mathcal{C}$. The curve is said to be irreducible, when the polynomial $f$ cannot be divided by a non-constant polynomial.

Note that when two polynomials define the same curve, they must be equal up to a scalar. For convenience and shorter formulation, we define a form $f \in \mathbb{C}[x, y, z]$ of degree $n$ to be an homogeneous polynomial in $x, y, z$ of total degree $n$.

\section{A.2.2 Tangency and singularities}

Let $\mathcal{C}$ be a curve of degree $d$ and let $\mathcal{L}$ be a given line. We can represent the line parametrically by taking two fixed points $\mathbf{a}$ and $\mathbf{b}$ on it, so that $\mathbf{a}$ general point $\mathbf{p}$ (except $\mathbf{b}$ itself) on it is given by $\mathbf{a}+\lambda \mathbf{b}$. The intersections of $\mathcal{L}$ and $\mathcal{C}$ are the points $\left\{\mathbf{p}_{\lambda}\right\}$, such that the parameters $\lambda$ satisfy the equation:

$$
J(\lambda) \equiv f\left(a_{x}+\lambda b_{x}, a_{y}+\lambda b_{y}, a_{z}+\lambda b_{z}\right)=0
$$

Taking the first-order term of the a Taylor-Lagrange expansion:

$$
\begin{aligned}
J(\lambda) & =f(\mathbf{a})+\lambda\left(\frac{\partial f}{\partial x}(\mathbf{a}) b_{x}+\frac{\partial f}{\partial y}(\mathbf{a}) b_{y}+\frac{\partial f}{\partial z}(\mathbf{a}) b_{z}\right) \\
& =f(\mathbf{a})+\lambda \operatorname{grad}_{\mathbf{a}}(f)^{T} \mathbf{b} \\
& =0
\end{aligned}
$$


If $f(\mathbf{a})=0$, $\mathbf{a}$ is located on the curve. Furthermore let assume that $\operatorname{grad}_{\mathbf{a}}(f)^{T} \mathbf{b}=0$, then the line $\mathcal{L}$ and the curve $\mathcal{C}$ meet at a in two coincident points. A point is said to be regular is $\operatorname{grad}_{\mathbf{a}}(f) \neq \mathbf{0}$. Otherwise it is a singular (or multiple) point. The set of singular points or singularities is denoted by $\operatorname{sing}(\mathcal{C})$. When the point a is regular, the line $\mathcal{L}$ is said to be tangent to the curve $\mathcal{C}$ at $\mathbf{a}$.

Proposition 19 The set of singularities $\operatorname{sing}(\mathcal{C})$ is finite.

Proof: A singular point a is such that: $f(\mathbf{a})=0$ and $\operatorname{grad}_{\mathbf{a}}(f)=0$. Thus it is located as the intersection of four distinct curves. Hence there are at most a finite number of such points.

Definition 7 Let $\mathcal{C}$ be a curve, defined by a polynomial $f$. Let $\mathbf{p}$ be a point on $\mathcal{C}$. The multiplicity of $\mathbf{p}$ is the smallest $m$ such as there exists $(i, j, k) \in \mathbb{N}^{3}$ with $i+j+k=m$ and:

$$
\frac{\partial^{m} f}{\partial^{i} x \partial^{j} y \partial^{k} z}(\mathbf{p}) \neq 0 .
$$

The multiplicity of $\mathbf{p}$ is denoted by $m(\mathbf{p}, \mathcal{C})$ or simply $m(\mathbf{p})$ if there is no ambiguity.

Note that $m(\mathbf{p}, \mathcal{C})=1$ if $\mathbf{p}$ is a regular point of $\mathcal{C}$. Otherwise $m(\mathbf{p}, \mathcal{C})>1$. If $m(\mathbf{p}, \mathcal{C})=2$, the point is called a double point, if $m(\mathbf{p}, \mathcal{C})=3$, a triple point, etc. If the point $\mathbf{p}$ is translated to the origin, then the affine part of the curve is given by the following polynomial:

$$
f_{\text {affine }}=f_{m}+f_{m+1}+\ldots+f_{d},
$$

where $f_{i}$ is a form of degree $d$ and $m$ the multiplicity of $\mathbf{p}$. Since $f_{m}$ is a form in two variables, we can write it as a product of linear factors $f_{m}=\prod l_{i}^{r_{i}}$, where $l_{i}$ are distinct lines. The lines $l_{i}$ are tangent to the curve at the singular point $\mathbf{p}$. A singular point is said to be ordinary if all its tangents are distinct. An ordinary double point is called a node.

Definition 8 Given a planar algebraic curve $\mathcal{C}$, the dual curve is defined in the dual plane, as the set of all lines tangent to $\mathcal{C}$ at simple points. The dual curve is algebraic and thus can be described as the set of lines $(u, v, w)$, that are the zeros of a form $\phi(u, v, w)=0$.

Proposition 20 Let $\mathcal{C}$ be a curve of degree d, which only singularities are nodes. The degree of the dual curve $\mathcal{D}$ is:

$$
m=d(d-1)-2(\# \text { nodes }),
$$

where (\#nodes) is the number of nodes.

\section{A.2.3 Inflexions points and Hessian curves}

We will also need to consider the notion of inflexion point:

Definition 9 An inflexion point a of a curve $\mathcal{C}$ is a simple point of it whose tangent intersects the curve in at least three coincident points. This means that the third order term of the Taylor-Lagrange development of $J(\lambda)$ must vanish too.

It will be useful to compute the inflexion points. For this purpose we define the Hessian curve $\mathcal{H}(\mathcal{C})$ of $\mathcal{C}$, which is given by the determinantal equation:

$$
\left|\frac{\partial^{2} f}{\partial x_{i} \partial x_{j}}\right|=0
$$

It can be proven (see [39]) that the points where a curve $\mathcal{C}$ meets its Hessian curve $\mathcal{H}(\mathcal{C})$ are exactly the inflexion points and the singular points. Since the degree of $\mathcal{H}(\mathcal{C})$ is $3(d-2)$, there are $3 d(d-2)$ inflexion and singular points counting with the corresponding intersection multiplicities (Bezout's theorem, see [39]).

\section{A.2.4 Genus and rational curves}

The genus of the algebraic curve can be defined in numerous manners. Some definitions are topological, some are analytic, and some are algebraic. For further details, the reader should consult [36]. Here it is sufficient to provide a partial definition of it.

Definition 10 For a planar algebraic curve, which degree is $d$ and which only singularities are nodes, the genus is defined as being the following number:

$$
g=\frac{(d-1)(d-2)}{2}-(\# \text { nodes }),
$$

where \#nodes is the number of nodes.

\section{A.3. Algebraic spatial curves}

An algebraic spatial curve is defined as being the intersection of two or more algebraic surfaces. In a more formal way, it is defined by a set of homogeneous equations:

$$
\mathbf{F}_{i}(X, Y, Z, T)=0
$$

In the body of this article, this representation is referred as the point-based representation. A point $\mathbf{P}=$ $[X, Y, Z, T]^{T}$ on the curve is said to be singular if: $\operatorname{grad}_{\mathbf{P}}\left(F_{i}\right)=0$ for all $i$. 


\section{A.3.1 Dual curve}

Let $X$ be an irreducible curve in $\mathbb{P}^{3}$, defined by the following family of polynomials: $\left\{F_{i}\right\}_{i}$. The dual curve $X^{*} \subset \mathbb{P}^{3 *}$ (where $\mathbb{P}^{3 *}$ is the dual projective threedimensional space) is a surface (see [18]) given by the polynomial $\Upsilon \in \mathbb{C}[A, B, C, D]$. The computation of $\Upsilon$ from $X$ is done by the following elimination problem:

Eliminate $X, Y, Z, \lambda_{i}$ from the following system:

$$
\begin{array}{cc}
F_{i}(X, Y, Z, 1) & =0 \\
{\left[\begin{array}{c}
A \\
B \\
C
\end{array}\right]-\sum \lambda_{i} \operatorname{grad}_{\mathbf{P}}\left(F_{i}(X, Y, Z, 1)\right)} & =0 \\
A X+B Y+C Z+D & =0,
\end{array}
$$

where $\mathbf{P}=[X, Y, Z]^{T}$. This system simply expresses the fact the tangent plane to the curve at a point $[X, Y, Z, 1]$ must be a linear combination of the gradients (that is the normals) of the surfaces defining the curve. The practical computation is done by an elimination engine, say Gröbner basis engine for instance $[5,12,13]$.

The conversion from the dual curve to the original curve is done by a similar elimination problem since the duality is an involution, that is the dual curve of the dual curve is the curve itself. Hence if $\Upsilon \in \mathbb{C}[A, B, C, D]$ is the polynomial defining the dual curve $X^{*} \subset \mathbb{P}^{3 *}$, the original curve $X$ is recovered as follows:

Eliminate $A, B, C, \lambda$ from the following system:

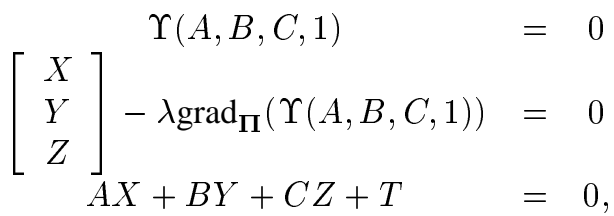

where $\Pi=[A, B, C]^{T}$.

\section{A.3.2 Curve representation in $\mathbb{G}(1,3)$}

It is well known that a line in $\mathbb{P}^{3}$ can be represented by its Plücker coordinates as point of $\mathbb{P}^{5}$ lying on special quadric, called the Grassmannian of lines of $\mathbb{P}^{3}$ and denoted by $\mathbb{G}(1,3)[3,10,18]$. Therefore we shall denote by $\mathbf{L}$ a line in $\mathbb{P}^{3}$ and by $\widehat{\mathbf{L}}$ its Plücker coordinates which makes it a point of $\mathbb{P}^{5}$. We proceed to show that a curve in $\mathbb{P}^{3}$ can be represented as a subvariety of $\mathbb{G}(1,3)$ which leads to very useful applications.

A smooth irreducible curve $X$ which degree is $d$ and embedded in $\mathbb{P}^{3}$ is entirely determined by the set of lines meeting it [18]. We define the following set of lines:

$$
\Lambda=\left\{\mathbf{L} \subset \mathbb{P}^{3} \mid \mathbf{L} \cap X \neq \emptyset\right\}
$$

The following facts are well known [18]:
1. $\Lambda$ is an irreducible subvariety of $\mathbb{G}(1,3)$.

2. There exists a homogeneous polynomial $\Gamma$, which degree is $d$, such that:

$$
Z(\Gamma) \cap \mathbb{G}(1,3)=\Lambda,
$$

where $Z(\Gamma)=\left\{\widehat{\mathbf{L}} \in \mathbb{P}^{5} \mid \Gamma(\widehat{\mathbf{L}})=0\right\}$.

3. $\Gamma$ is defined modulo the $d$-th graded piece of the ideal defining $\mathbb{G}(1,3)$, that is modulo $I(\mathbb{G}(1,3))_{d}$.

4. The dimension of the $d$-th graded piece of the homogeneous coordinate ring of $\mathbb{G}(1,3)$, that is of $S(\mathbb{G}(1,3))_{d}$ is for $d \geq 2$ :

$$
N_{d}=\left(\begin{array}{c}
d+5 \\
d
\end{array}\right)-\left(\begin{array}{c}
d-2+5 \\
d-2
\end{array}\right)
$$

5. It is sufficient to pick $N_{d}$ generic points on $\Lambda$ to find $\Gamma$ modulo $I(\mathbb{G}(1,3))_{d}$. Each such point $\widehat{\mathbf{L}}$ yields one linear equation on $\Gamma$ :

$$
\Gamma(\widehat{\mathbf{L}})=0
$$

Definition 11 Any element of the equivalence class of $\Gamma$ is said to be the Chow polynomial of the curve $X$.

The previous properties provides us with a way to compute $\Gamma$ from a set of discrete measurements extracted from image sequences. However we proceed to show, how one can recover $\Gamma$ from the usual point based representation of the curve. Let $\left\{F_{i}\right\}$ the family of polynomials defining the curve. The computation of $\Gamma$ is done as follows:

Eliminate $X, Y, Z$ from the following system:

$$
\begin{aligned}
& F_{i}(X, Y, Z, 1)=0 \\
& \widehat{\mathbf{L}} \vee\left[\begin{array}{c}
X \\
Y \\
Z \\
1
\end{array}\right]=0,
\end{aligned}
$$

where $\widehat{\mathbf{L}}=\left[L_{1}, L_{2}, L_{3}, L_{4}, L_{5}, L_{6}\right] \in \mathbb{P}^{5}$ represents a line meeting the curve and $\vee$ is the join operator (see $[3,10]$ ). The join of $\widehat{\mathbf{L}}$ and the point $\mathbf{P}=[X, Y, Z, 1]^{T}$ vanishes to express the fact that $\mathbf{P}$ lies on the line represented by $\widehat{\mathbf{L}}$.

When given $\Gamma$ as a result of the previous elimination, we shall compute its normal form (to get a canonical representation of $\Gamma$ ) with respect to the equation defining $\mathbb{G}(1,3)$ :

$$
L_{1} L_{6}-L_{2} L_{5}+L_{3} L_{4}=0
$$

Given the Chow polynomial, it is easy to obtain the point-based representation. Let $\Gamma$ be the Chow polynomial of the curve. Follow the following procedure: 
1. Pick three generic points, $Q_{1}, Q_{2}, Q_{3}$ on the plane at infinity (last coordinate zero).

2. Consider a point $P=[X, Y, Z, 1]^{T}$ in the affine piece of $\mathbb{P}^{3}$. The point $P$ is lying on $X$ if any linear combination of the three lines $\left(P Q_{i}\right)_{i=1,2,3}$ is a zero of the Chow polynomial. This yields $\left(\begin{array}{c}d+2 \\ d\end{array}\right)$ equations defining completely the curve $X$. 